\title{
Introducing Along-Track Error Correlations for Altimetry Data in a Regional Ocean Prediction System
}

\author{
Andrea Storto, Paolo Oddo, Elisa Cozzani, And Emanuel Ferreira Coelho ${ }^{\mathrm{a}}$
}

Centre for Maritime Research and Experimentation, La Spezia, Italy

(Manuscript received 26 November 2018, in final form 29 April 2019)

\begin{abstract}
Because of the systematic error in the processing of altimetry data, sea level anomaly (SLA) observation errors are likely affected by nonnegligible spatial correlations. To account for these, we exploit the synergy of altimetry data with in situ profiles from gliders, piloted to follow the altimetry tracks during the Long-Term Glider Mission for Environmental Characterization 2017 (LOGMEC17) observational campaign in the Ligurian Sea. The assimilation of along-track unfiltered sea level anomalies in a regional ocean analysis and forecast system is consequently optimized by means of introducing spatial correlations for the SLA observation errors. In particular, collocated data of glider and altimetry are used to derive an along-track error covariance model for the sea level anomaly assimilation, assuming that most of the covariance behavior versus separation distance stems from altimetry. Spatial scales of the altimetry error are found to have a correlation radius of about $12 \mathrm{~km}$ for the dataset utilized in the Ligurian Sea, using a simple Gaussian shape for the error correlation, shorter than the correlation radius found through assimilation output diagnostics. A variational data assimilation system is modified to relax the usual assumption of uncorrelated altimetry observation errors, thus allowing for along-track error correlations. Its implementation provides promising results in the regional ocean prediction system, outperforming in most verification skill scores the use of uncorrelated observational errors without compromising the analysis scheme efficiency.
\end{abstract}

\section{Introduction}

Satellite altimetry has revolutionized our knowledge of the ocean dynamics since more than two decades through systematic provision of information on both eddy and finescale dynamics (Fu et al. 2010; Morrow et al. 2017) and large basin-scale circulation (Stammer 1997). Consequently, the impact of altimetry observations has proven dramatic in both global (Verrier et al. 2017) and regional (Pujol et al. 2010) operational analysis and forecast systems, representing a crucial observing network complementary to in situ observation profiles (Storto et al. 2013).

Assimilating altimetry observations contains however a number of difficulties: the definition of the multivariate balances in the data assimilation system determines the vertical projection of the altimetry innovations in the subsurface and represents a crucial choice in operational

\footnotetext{
${ }^{a}$ Current affiliation: Ocean Acoustic Services and Instrumental Systems, Lexington, Massachusetts.
}

Corresponding author: Andrea Storto, andrea.storto@cmre.nato.int systems. Such a choice also depends on the spatial scales and dynamical regime of the study region, along with technical issues linked for instance to the computational costs of the assimilation scheme. Moreover, the observation error characterization is in general difficult, because not only the instrumental but also the representation errors and those associated with the geophysical corrections (e.g., Storto et al. 2011) shall be assessed. Finally, in spite of recent advances in gravimetric missions, the definition of the mean dynamic topography (MDT) for satellite data assimilation (i.e., the steady level of the sea surface shaped by the persistent ocean circulation), needed to compare sea level anomalies to modeled sea surface height fields, is nontrivial to estimate. This is mostly due to the low resolution of gravimetry data compared to regional ocean model implementations, thus requiring supplementary information; furthermore, the intrinsic misrepresentation of several physical processes by ocean models (e.g., Vidard et al. 2009) may complicate the assessment of optimal MDT for data assimilation.

To ease the cost function formulation and minimization, most variational data assimilation systems assume that any pair of observations has errors mutually 
uncorrelated; that is, no observation error correlation is allowed. This leads to defining the observation-error covariance matrix as a diagonal matrix, with obvious advantages for the variational algorithm implementation and the minimization preconditioning, namely the speed of convergence. However, one may question such assumption for high-resolution satellite observations (Fowler et al. 2018). In particular, satellite altimetry data undergo a number of geophysical corrections (e.g., tropospheric, ionospheric, tidal; see Chelton et al. 2001) that together with instrumental errors in the radar sensor onboard the satellites and cross-satellite calibration, make the assumption of uncorrelated altimetry observational errors likely exaggerated. With the increase of resolution of future altimeters, spatial correlations are likely to become less negligible (Gaultier et al. 2016).

Usual reliefs consist in satellite data combination (superobservations; e.g., Oke et al. 2008), thinning or inflation of observational errors (e.g., Liu and Rabier 2003; Rainwater et al. 2015). High-resolution regionalscale analysis systems may suffer from this assumption more than coarse-resolution systems, provided that their resolution is higher than the satellite data, and thus observation thinning procedures may prevent extracting the eddy-scale information from altimetry observations. Relaxing such assumption is the main goal of this article, together with the estimation of the correlation length scale itself, which is here performed through exploiting the collocation of altimetry tracks with glider tracks that have been synchronized with the footprints of simultaneous altimeter tracks during the Long-Term Glider Mission for Environmental Characterization 2017 (LOGMEC17) observational campaign in the Ligurian Sea.

Previous attempts of introducing altimetry error correlations proved promising. Brankart et al. (2009, 2010) showed that applying a linear transformation to the observation vector and augmenting the observation vector with gradient observations to introduce error correlations improves the ocean circulation in regional prediction systems. A similar strategy has also been explored by Ruggiero et al. (2016) to investigate the optimal assimilation of Surface Water and Ocean Topography (SWOT) simulated altimetry data. Again with the aim of optimally assimilating SWOT data, Yaremchuk et al. (2018) propose an approximated expression for the observation error covariance matrix through parametric polynomials. All these studies indicate that correlated errors in altimetry observations should be accounted for in the formulation of the data assimilation problem, which is the main focus of this article.
The structure of the paper is as follows: After this introduction, section 2 briefly introduces the observational data used in this study, and the regional analysis and forecast system. Section 3 provides a novel formulation and assessment of length scales for assimilating altimetry observations with correlated alongtrack errors. Section 4 provides the assessment of the impact of the correlation-aware formulation of the altimetry assimilation. Finally, section 5 discusses the main achievements and conclusions.

\section{Data and methods}

\section{a. Observational datasets}

The LOGMEC17 took place in the Ligurian Sea from 13 September to 14 November 2017. The main objective of the LOGMEC17 sea trial is to carry out a synergic study of the variability and predictability of the acoustical and oceanographic environments (Borrione et al. 2019). Two oceanographic deep gliders were deployed on 21 September and continuously monitored the Ligurian Sea until 12 November 2017 when they were recovered. Glider missions were designed to optimally synchronize the gliders tracks with satellite altimeter passages.

The Copernicus Marine Environment Monitoring Service (CMEMS) provides a large number of satellite products (Le Traon et al. 2017). In the present study we focus on the sea level anomaly (SLA) dataset, presented by Pujol et al. (2016). For the purposes of the present study the Tailored Altimetry Products for Assimilation System (TAPAS) dataset were used. SLA data in the TAPAS dataset are provided without the usual along-track subsampling, therefore allowing for a full horizontal resolution of about $7 \mathrm{~km}(1 \mathrm{~Hz})$. See also Dufau et al. (2016) for further details. The leadingedge footprint, namely "the surface of the sea that is illuminated when the backscattered energy increases on the first nonzero waveform gates" (Dibarboure et al. 2014), which drives the signal resolution (e.g., Quartly 1998), is on the order of $2-3 \mathrm{~km}$, depending on the satellite orbit height and significant wave height (Chelton et al. 1989; Cheney 2001). The unfiltered version of the sea level anomalies are considered here, which proved to adequately sample the short scales (Wunsch and Stammer 1998). The dataset used is the one released in near-real time, which in turns makes use of the European Centre for Medium-Range Weather Forecasts (ECMWF) operational analyses for the dynamic atmospheric corrections (Taburet et al. 2018), at a resolution of about $9 \mathrm{~km}$.

All the tracks available in the near-real-time dataset for the period of the sea trial are shown in Fig. 1 and are 


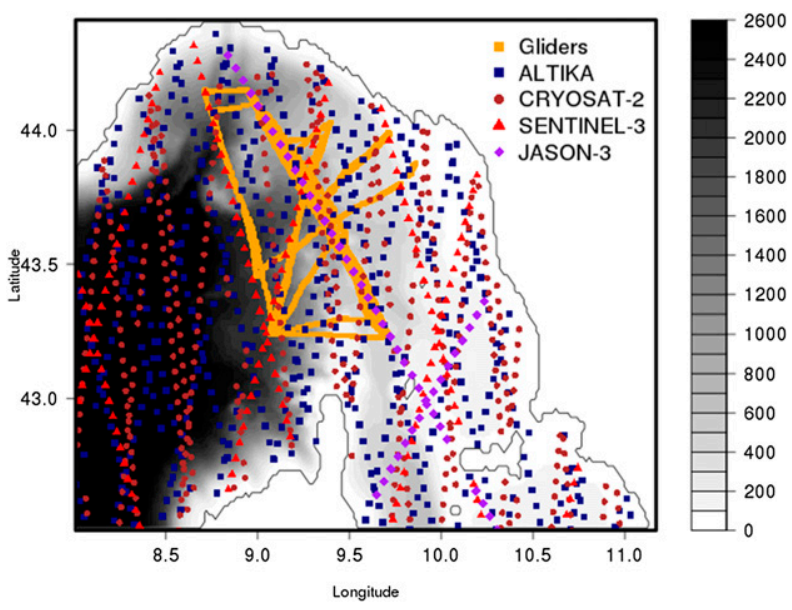

FIG. 1. Observational data used in this study. The geographical area in the picture corresponds to the Ligurian Sea ocean model domain. Contours represent the bathymetry used in the model $(\mathrm{m})$ and derived from GEBCO.

those of satellites Satellite with Argos and AltiKa (SARAL), CryoSat-2, Jason-3, and Sentinel-3. Details on the altimetry products and previous assimilation studies are provided in Oddo et al. (2016). The glider sampling is mostly located in correspondence of two altimetry tracks from Jason-3 and Sentinel-3 (the two altimetry satellites with repeated orbit among the available constellation). Table 1 reports the dates and the satellite of the glider tracks synchronized with the altimetry tracks.

\section{b. The Ligurian Sea regional analysis and forecast system}

The analysis and forecast system implemented for the experiments that make use of LOGMEC17 sea-trial observations is based on a regional implementation of the NEMO primitive equation model, version 3.6 (Madec et al. 2012), built upon the previous modeling experience at CMRE (Oddo et al. 2016). The NEMO domain covers the Ligurian Sea with two open boundaries on the western and southern sides located at $8^{\circ} \mathrm{E}$ and $42.5^{\circ} \mathrm{N}$, respectively (Fig. 1). The bathymetry is taken from General Bathymetric Chart of the Oceans (GEBCO; Weatherall et al. 2015). The horizontal resolution of the model is about $1.8 \mathrm{~km}$ and the vertical grid is discretized using 91 depth levels with partial steps (Barnier et al. 2006). Surface fluxes are calculated iteratively every model time step $(100 \mathrm{~s})$ by means of bulk formula using the atmospheric fields of air temperature, humidity, winds and cloud cover (Pettenuzzo et al. 2010) from the $1 / 4^{\circ}$-resolution atmospheric operational analyses provided by the National Centers for Environmental Predictions (NCEP; available at
TABLE 1. Satellites and dates of the altimetry tracks collocated with the glider tracks for use in the altimetry error correlation length scale of section 3 .

\begin{tabular}{cc}
\hline \hline Satellite & Dates of glider collocated tracks \\
\hline Jason-3 & 26 Sep 2017 \\
& 6 Oct 2017 \\
& 15 Oct 2017 \\
& 4 Nov 2017 \\
Sentinel-3 & 2 Oct 2017 \\
& 3 Oct 2017 \\
& 29 Oct 2017 \\
& 30 Oct 2017
\end{tabular}

https://nomads.ncep.noaa.gov). Ocean forecasts are produced using the atmospheric forecasts from NCEP. The NEMO model is one-way nested with the CMEMS Mediterranean Sea operational model (the Mediterranean Forecasting System; Oddo et al. 2014) that provides the lateral boundary conditions (LBCs). The satellite level4 SST product from CMEMS (Buongiorno Nardelli et al. 2013) is ingested into the model through the adjustments of surface heat flux by means of a Newtonian relaxation algorithm. The relaxation coefficient is equal to $-60 \mathrm{~W} \mathrm{~m}^{-2} \mathrm{~K}^{-1}$ at nighttime, and gently decreases to $-10 \mathrm{~W} \mathrm{~m}^{-2} \mathrm{~K}^{-1}$ at noon because of the use of nighttime measurements only in the production of the SST analysis.

The NEMO model is coupled with a three-dimensional variational data assimilation (3DVAR) system, which uses the standard incremental formulation and is derived by the variational code of Storto et al. (2011), in turn adapted from Dobricic and Pinardi (2008). The analysis system assimilates all available altimetry and in situ profile observations, the latter after "superobbing" the data (i.e., averaging over each model grid cell). The analysis increments $\delta \mathbf{x}$ (defined as analysis minus background) are found for the ocean state $\mathbf{x}$ at the minimum of the following cost function:

$J(\delta \mathbf{x})=\frac{1}{2} \delta \mathbf{x}^{\mathrm{T}}(\mathbf{B})^{-1} \delta \mathbf{x}+\frac{1}{2}(\mathbf{H} \delta \mathbf{x}-\mathbf{d})^{\mathrm{T}} \mathbf{R}^{-1}(\mathbf{H} \delta \mathbf{x}-\mathbf{d})$,

where $\mathbf{B}$ and $\mathbf{R}$ are the background- and observationerror covariance matrices, $\mathbf{d}$ is the vector of innovations (or misfits), with $\mathbf{d}=\mathbf{y}-H\left(\mathbf{x}^{b}\right), \mathbf{y}$ the observation vector, $\mathbf{x}^{b}$ the background, $H(\cdot)$ the observation operator mapping the ocean state onto observation space, and $\mathbf{H}$ the tangent-linear version of the observation operator, linearized around the background state. This implementation considers the first guess at appropriate time (FGAT) algorithm; namely, $\mathbf{d}$ is computed using the background fields at the exact time of the observations. To precondition the minimization problem and avoid the inversion of $\mathbf{B}$, a control vector 
(v) transformation is applied, so that the cost function actually minimized is

$$
J(\mathbf{v})=\frac{1}{2} \mathbf{v}^{\mathrm{T}} \mathbf{v}+\frac{1}{2}(\mathbf{H} \mathbf{V} \mathbf{v}-\mathbf{d})^{\mathrm{T}} \mathbf{R}^{-1}(\mathbf{H V} \mathbf{v}-\mathbf{d}),
$$

with

$$
\delta \mathbf{x}=\mathbf{V} \mathbf{v} ; \quad \mathbf{B}=\mathbf{V} \mathbf{V}^{\mathrm{T}} .
$$

The operator $\mathbf{V}$ is thus the left square root of $\mathbf{B}$, and it is defined as a sequence of operators:

$$
\mathbf{V}=\mathbf{V}_{b} \mathbf{V}_{h} \mathbf{V}_{v}
$$

where $\mathbf{V}_{v}$ and $\mathbf{V}_{h}$ account for the vertical covariances and horizontal correlations, modeled through multivariate empirical orthogonal functions (EOFs) and the application of a recursive filter operator, respectively. The operator $\mathbf{V}_{b}$ accounts for cross covariances, that is, balances between state variables. Here, we use a formulation similar to Weaver et al. (2005), where a sea level balanced component, provided by the dynamic height formula (proportional to the vertically integrated negative density anomalies, from a depth of $400 \mathrm{~m}$ to the sea surface), is combined with an unbalanced component from the statistics of the residuals of the balance.

Background-error vertical covariances (EOFs) were estimated from the dataset of temperature, salinity and sea level anomalies with respect to the long-term mean (from September to November 2017), taken as daily means every 3 days to ensure ergodicity of the statistics. In situ observation error profiles were scaled from misfit statistics collected in a preliminary model simulation. The temperature errors are found to peak around the mixed layer depth (where temperature vertical gradients are maxima) and the salinity around the freshwater intrusion depth and the halocline (not shown). Both sea level anomaly and in situ data undergo a background-quality check, prior to the variational quality control implemented as in Storto (2016), namely observations with a departure from the background larger than thrice the squared sum of observation and background error variances are rejected. The MDT for SLA data assimilation is provided by Rio et al. (2014). SLA data are assimilated only in areas deeper than $400 \mathrm{~m}$, for consistency with the dynamic height formula. Note that the dynamic height formula used by Rio et al. (2014) for the MDT calculation integrates the density anomalies in the top $350 \mathrm{~m}$, while our balance operator in the top $400 \mathrm{~m}$. We have verified that such a difference in the integration limits is negligible, namely the area-averaged explained variance of the $350-400-\mathrm{m}$ layer with respect to the $0-400-\mathrm{m}$ one is equal to $1.8 \%$.

\section{Along-track observation error correlations}

In this section, we reformulate the variational cost function to account for along-track observational error correlations. The great majority of data assimilation systems assume for simplicity that observation errors are mutually uncorrelated. Relaxing such assumption thus implies introducing off-diagonal terms in the observation-error covariance matrix. The main drawback in constructing $\mathbf{R}$ with off-diagonal terms is the possible deterioration of the convergence speed, as a nondiagonal $\mathbf{R}$ reduces the benefits of the usual $\mathbf{B}$-based preconditioning of the variational minimization problem (e.g., Weston 2011). Furthermore, an explicit inversion of $\mathbf{R}$ is required; this might become prohibitive for a large size of the observation vector.

There exist different strategies to account for correlated observation errors, which include the inversion of $\mathbf{R}$ through eigendecomposition, tridiagonal definition of $\mathbf{R}$, or reformulating the variational problem in the dual space to precondition the minimization problem with respect to $\mathbf{R}$ and not B [see Stewart et al. (2013) for a discussion of possible strategies]. All these solutions may have different relative merits, depending on the specific application (domain size and observation count).

\section{a. Formulation}

Here, we consider that only altimetry observations belonging to the same track may have correlated observational errors, which in turn depend on their distance and a prescribed correlation length scale. Thus, only a few off-diagonal terms of $\mathbf{R}$ are nonzero, mostly around the diagonal. Provided that the total number of observations in the regional analysis system developed for LOGMEC17 is small, the explicit inversion of $\mathbf{R}$ becomes feasible. To account for correlated altimetry observation errors, the variational cost function is redefined as

$$
\begin{aligned}
J(\delta \mathbf{x})= & \frac{1}{2} \delta \mathbf{x}^{\mathrm{T}}(\mathbf{B})^{-1} \delta \mathbf{x}+\frac{1}{2}\left(\mathbf{H}_{i} \delta \mathbf{x}-\mathbf{d}_{i}\right)^{\mathrm{T}} \mathbf{R}_{i}^{-1}\left(\mathbf{H}_{i} \delta \mathbf{x}-\mathbf{d}_{i}\right) \\
& +\frac{1}{2} \sum_{k=0}^{N}\left(\mathbf{H}_{k} \delta \mathbf{x}-\mathbf{d}_{k}\right)^{\mathrm{T}} \mathbf{R}_{k}^{-1}\left(\mathbf{H}_{k} \delta \mathbf{x}-\mathbf{d}_{k}\right)
\end{aligned}
$$

where we break the observational cost function in terms accounting separately for in situ observations and for the $N$ satellite tracks. For sake of clarity, the control variable transformation is not shown in Eq. (5). The first two rhs terms are the usual cost function terms penalizing against the background and the in situ observations, respectively, with $\mathbf{R}_{i}$ the diagonal in situ observationerror covariance matrix, the subscript $i$ standing for in situ. The third term in the rhs penalizes against 
the altimetry observations, grouped in the $N$ tracks. For each $k$ th track, an observation-error covariance matrix $\mathbf{R}_{k}$ can be defined in general as (see, e.g., Brankart et al. 2009)

$$
\mathbf{R}_{k}=\mathbf{O}_{k}+\mathbf{N}_{k}=\mathbf{O}_{k}+\mathbf{D}_{k}^{1 / 2} \mathbf{C}_{k} \mathbf{D}_{k}^{1 / 2},
$$

where $\mathbf{O}_{k}$ and $\mathbf{N}_{k}$ are the uncorrelated and correlated components of $\mathbf{R}_{k}$, respectively. While $\mathbf{O}_{k}$ is a diagonal matrix, $\mathbf{N}_{k}$ is further decomposed, with $\mathbf{D}_{k}$ the diagonal matrix containing the observation-error variances (correlated component only), and $\mathbf{C}_{k}$ the correlation matrix, where the generic element $(i, j)$ has the Gaussian form $C_{i, j}=\exp \left(-d_{i, j}^{2} / 2 L^{2}\right)$, with $d_{i, j}$ the distance between the observations at locations $i$ and $j$, and $L$ the altimetry observation-error correlation length scale. Practical advantages of implementing the along-track correlation, with respect to the case where the entire $\mathbf{R}$ is nondiagonal, are that (i) the dimension of $\mathbf{R}_{k}$ is small and allows for fast inversion; (ii) the off-diagonal terms are only a few and possibly do not impact the speed of the minimization convergence; and (iii) we specifically account only for intratrack error correlations, the most conceptually important ones, without introducing spurious error correlations.

Haben et al. (2011) and El Akkraoui et al. (2013) discuss how the control variable transformation preconditioning [Eq. (2)] prevents minimization problems when the number of observations is much smaller than the size of the control vector, and the background-error correlation length scales dominate the minimization problem. In our configuration, the number of observations leading to off-diagonal terms for $\mathbf{R}_{k}$ is on the order of 10-30 per assimilation cycle (observations on the same track), against a much larger size for the control vector of $\mathbf{v}\left(\sim 1 \times 10^{6}\right)$. Indeed, the Hessian of the cost function, after the control variable transformation, is given by $\mathbf{I}_{v}+\mathbf{V}^{\mathrm{T}} \mathbf{H}^{\mathrm{T}} \mathbf{R}^{-1} \mathbf{H V}$, where $\mathbf{I}_{v}$ is the identity matrix of size equal to that of the control vector. The second term (background term) is not full rank because of the much smaller size of observation vector compared to the control vector size, but the Hessian matrix is full rank, with unity as the smallest eigenvalue. Therefore the impact of the uncorrelated altimetry error on the preconditioning is expected to be very small with the regional configuration used. However, global applications at moderate resolution (with large number of observations and relatively small control vector size) may suffer from suboptimal preconditioning in our formulation. The inversion of the $\mathbf{R}_{k}$ matrices [Eq. (5)] is obtained through computing the Moore-Penrose pseudoinverse by means of singular value decomposition (SVD) (Golub and Van Loan 2012, p. 290).

To provide a visual example, Fig. 2 shows the altimetry observation-error covariance matrix for a case where

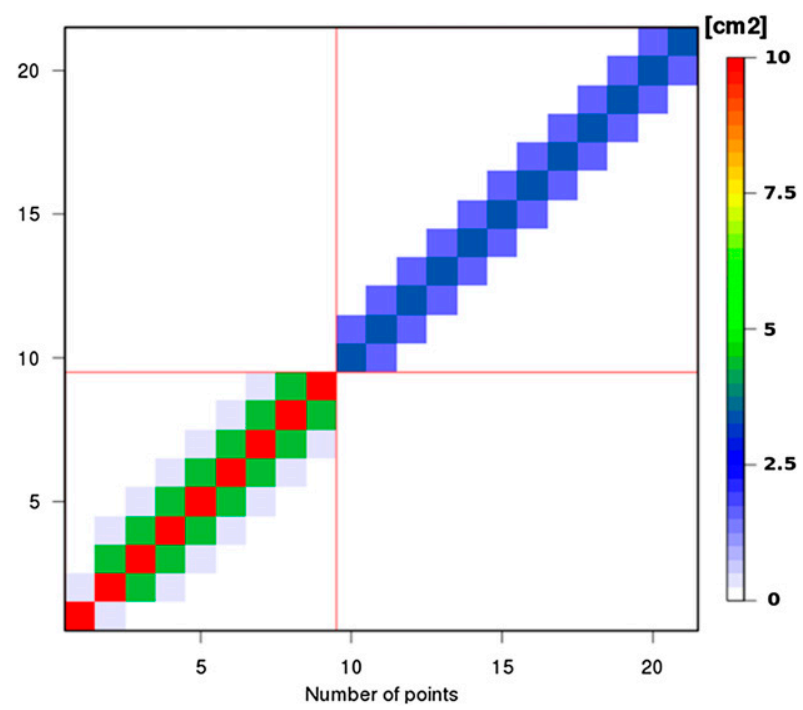

FIG. 2. Example of $\mathbf{R}$ matrix when two altimetry tracks are available in the analysis system and along-track error correlations are allowed. Red lines indicate the separation between the two tracks in the matrix; the error correlations between observations belonging to different tracks are zero by construction [Eq. (5)].

only two tracks are available. The red rectangles show the tracks. Off-diagonal terms are close to the diagonal ones, namely the matrix is close to a tridiagonal matrix. Moreover, considering that the distance between two consecutive pairs of observations is approximately constant along a satellite track, and imposing a homogeneous correlation length scales, such structure is close to the Markov matrix proposed by Stewart et al. (2013). Intertrack error correlations are zero by construction.

\section{b. Estimation of error correlations}

Allowing intratrack observational errors requires the estimation of the correlation length scales. However, this is nontrivial, because several factors concur to correlated altimetry noise, and we refer here to the data actually ingested in our system (i.e., real-time alongtrack unfiltered sea level anomalies). Using unfiltered data allows to sample small spatial scales (Wunsch and Stammer 1998; Backeberg et al. 2014), and the impact of aliasing is reduced (Byrne and McClean 2008), although small-scale noise may be spatially correlated. Rapid changes of backscatter power due to atmospheric and surface events may induce systematic errors at small scales (typically on the order of $30 \mathrm{~km}$; Dibarboure et al. 2014). Dynamic atmospheric correction, whose correlation is related to the atmospheric analysis used, depend in turn on the specific error correlation length scales inherited by the atmospheric model (i.e., the ECMWF operational analyses) performed at about $9 \mathrm{~km}$ of horizontal resolution. While error variances 
may be similar between real-time and delayed-time altimetry data that use operational analyses and reanalyses, respectively (e.g., Carrere et al. 2016), their error spatial scales may differ significantly. For instance, wet troposphere correction errors found through the SWOT simulator were shown to have short length scales (tenths of a kilometer) in both cross- and alongtrack directions at midlatitudes (Ruggiero et al. 2016). Ocean tide models may also lead to spatially correlated errors within the tidal correction procedure: the resolution of such models is on the order of $10-20 \mathrm{~km}$ and their error structures strongly depend on the specific location (Stammer et al. 2014). Finally, the mean dynamic topography may also introduce error structures, linked to its resolution (about $7 \mathrm{~km}$ for the SMDTMED-2014; Rio et al. 2014) and to the use of drifter velocities in its computation. All these factors contribute to altimetry error correlated in space. For instance, in the mapping methodology used for L4 data (Pujol et al. 2016), error length scales are assumed to be about $35 \mathrm{~km}$ [using a Gaussian correlation function fitted to the actual correlation function used in Pujol et al. (2016)]. Analyzing the different contribution of these factors on the resulting length scale is extremely complicated, because of the lack of reference data for each of the corrections applied to altimetry data, and it is beyond the scope of this work, where the aim is to estimate the resulting length scale for use in data assimilation systems. It is also possible that the different altimetric satellites may be characterized by different error correlation length scales, or that the length scales vary locally, although we assume for sake of simplicity and because of lack of enough collocated data that the length scale is spatially homogeneous and is the same for all satellites.

Estimating the altimetry error correlation length scale can be done for instance through analysis of the assimilation output statistics (so-called Desroziers's method; Desroziers et al. 2005) applied to pairs of observations (Bormann and Bauer 2010). This approach is relatively straightforward with a running analysis system, but it may lead to inaccurate results when background and observation error correlation length scales are comparable (Waller et al. 2016), which is actually the case for regional models, or when the prescribed values of both errors are not accurate enough. For these reasons, we prefer to use a separation method inspired by Hollingsworth and Lönnberg (1986) and originally proposed to estimate background-error covariances, and exploit the collocations between altimetry and glider data. Previous studies suggest that the two observing networks are able to sample the same sea level patterns (Morrow et al. 2017; Borrione et al. 2019).

First of all, consider for a generic location $i$ the dynamic height formula that diagnoses anomalies of sea level from profiles of temperature and salinity:

$$
\mathrm{DH}_{i}=-\frac{1}{\rho_{0}} \int_{z_{l}}^{0} \delta \boldsymbol{\rho}_{i}(z) d z
$$

with $\rho_{0}$ the reference density $\left(1026 \mathrm{Kg} \mathrm{m}^{-3}\right), z_{l}$ the bottom depth for integration (also called level of no motion), which is set equal to $200 \mathrm{~m}$ of depth after preliminary tests, and $\delta \boldsymbol{\rho}_{i}(z)$ the density anomaly, referenced to the three-dimensional long-term mean density (as in Storto et al. 2017) from a previous NEMO experiment. The use of the dynamic height is widely spread especially in data assimilation contexts (e.g., Weaver et al. 2005) and it represents a linearized version of the Cooper and Haines (1996) scheme where the SSH increments depend on the lifting and lowering of the water column. Note that the depth used in Eq. (7) is shallower than that used in the sea level balance operator within the variational analysis (200 and $400 \mathrm{~m}$, respectively), in order to use as many collocated glider profiles as possible while preserving the representation of baroclinic structures (Borrione et al. 2019).

If one considers that the difference $d_{i}$ between SLA and glider-derived sea level [through the dynamic height formula; Eq. (7)] at location $i$ is equal to the difference of their errors:

$$
d_{i}=\mathrm{SLA}_{i}-\mathrm{DH}_{i}=e_{i}^{\mathrm{SLA}}-e_{i}^{\mathrm{DH}},
$$

where the term $e_{i}^{\mathrm{DH}}$ also includes errors due to the inaccuracies of the dynamic height formula, it is then possible to calculate the covariance of $d$ as a function of the distance (viz., between locations $i$ and $j$ ). If error correlations between SLA and glider data are assumed to be zero, and spatial correlations of glider data are assumed to be negligible (or nonnegligible only for very small separation distances), then the resulting covariance will be equal to the SLA spatial covariance (for $i \neq$ $j$ ), while for $i=j$, there will also be the glider error variance contribution; namely,

$$
\begin{array}{ll}
\mathbf{E}\left[\left(d_{i}\right)^{\mathrm{T}}\left(d_{j}\right)\right] \cong \mathbf{E}\left[\left(e_{i}^{\mathrm{SLA}}\right)^{\mathrm{T}}\left(e_{j}^{\mathrm{SLA}}\right)\right] & \text { for } \quad i \neq j \\
\mathbf{E}\left[\left(d_{i}\right)^{\mathrm{T}}\left(d_{j}\right)\right] \cong \mathbf{E}\left[\left(e_{i}^{\mathrm{SLA}}\right)^{\mathrm{T}}\left(e_{j}^{\mathrm{SLA}}\right)\right]+\mathbf{E}\left[\left(e_{i}^{\mathrm{DH}}\right)^{\mathrm{T}}\left(e_{j}^{\mathrm{DH}}\right)\right] & \text { for } \quad i=j
\end{array}
$$


where $\mathbf{E}[\cdot]$ is the expectation operator. Note that the assumption of spatially uncorrelated errors for glider tracks is in practice difficult to verify because of the lack of independent data. Nevertheless, we can reasonably assume that most of the spatial error covariance signal stems from the procedures used in the processing of altimetry data, thus justifying such assumption.

Using Eq. (9), it is possible to fit the empirical covariance curve to find the SLA error correlation length scale $L$. Such a computation was performed using eight satellite tracks under which gliders were piloted (Table 1), for a total of 111 collocated satellite observations and glider profiles. Glider profiles may reach depths up to $1000 \mathrm{~m}$, with $525 \mathrm{~m}$ of depth being the averaged maximum depth of the profiles. The distance between adjacent profiles is on the average equal to $1.2 \mathrm{~km}$. Prior to the computation, dynamic height estimates from glider profiles [using Eq. (7)] were spatially averaged in order to approach the same horizontal resolution of the altimetric satellites, which is approximately $6.5 \mathrm{~km}$ at these latitudes. The average is performed on separate bins, in order not to introduce any spurious correlations in the gliderderived sea level data.

Results of the assessment are shown in Fig. 3. In particular, the top panel shows the estimated curve (black line) and the fitted curve. To consider the increase of covariance near the zero separation distance, the curve is assumed to be a linear combination of two Gaussian curves, which after the fitting are characterized by values of 3 and $12 \mathrm{~km}$, respectively, for the Gaussian length scales. The fitting also indicates that each of the two curves, representing the uncorrelated and correlated components, respectively, counts for $50 \%$ of the covariance: the two fitted functions are both multiplied by $4 \mathrm{~cm}^{2}$ (collocation variance) to obtain the estimated curve. The panel also reports the same estimates obtained through the consistency diagnostics of the Desroziers's method (Desroziers et al. 2005). These estimates are obtained through assimilation output diagnostics performed on sea level anomaly innovations and residuals from the experiment Rdiag (the one assimilating altimetry and glider data with uncorrelated observation errors, see section 4). Formally, the diagnosed $\tilde{\mathbf{R}}_{k}$ is equal to $\tilde{\mathbf{R}}_{k}=\mathbf{E}\left[\left(\mathbf{y}-\mathbf{H} \mathbf{x}^{a}\right)\left(\mathbf{y}-\mathbf{H} \mathbf{x}^{b}\right)^{\mathrm{T}}\right]$. These diagnostics indicate a small covariance near the zero separation distance, likely due to the smaller error when only SLA data are used, confirming that dynamic height estimates from glider augment the error variance. Nevertheless, spatial scales appear longer than the collocation method. Desroziers's diagnostic may indeed produce wrong estimates of the true error covariances in the case of nonoptimal error statistics in
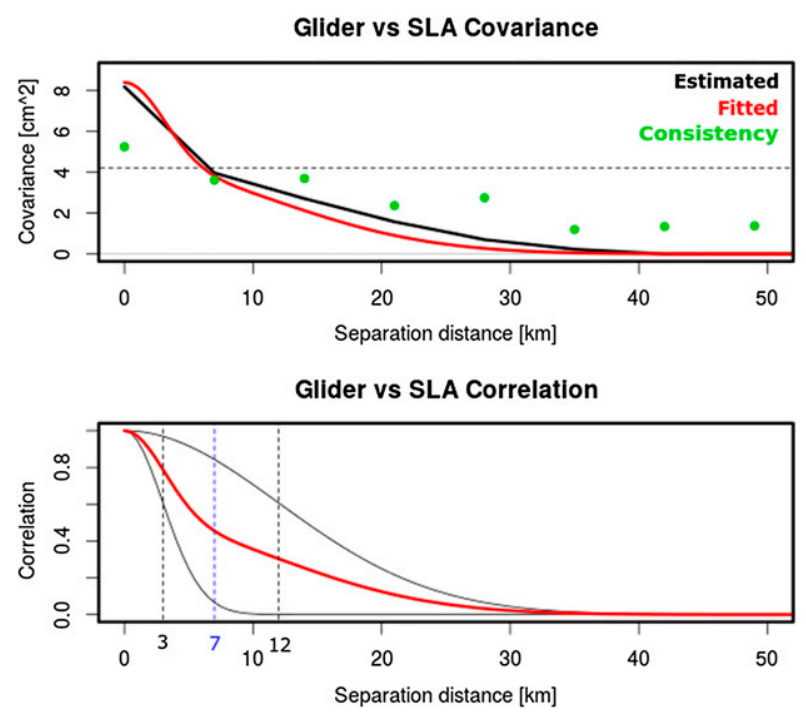

FIG. 3. Estimated along-track error correlation. (top) Estimated covariances as a function of separation distance (black) and corresponding fitted curve (red). Values obtained from consistency diagnostics (Desroziers et al. 2005) are shown as green points. (bottom) Fitted correlation as a function of separation distance. The two black lines represent the Gaussians from the fitting, while the red thick line represents the resulting correlation function. Dashed vertical black lines are correspond to the two Gaussian correlation length scales, while the dashed vertical blue line corresponds to the average spatial resolution of altimetry data in the study region.

the assimilation (Michel 2018). Furthermore, Desroziers et al. (2010) state that the correlation lengths in $\mathbf{B}$ and $\mathbf{R}$ should be different enough for the diagnostic to provide useful results. Waller et al. (2016) suggest that, when $\mathbf{R}$ is treated as uncorrelated in the assimilation (which is often the case), the diagnostic computation may lead to wrong results. Therefore, the longer spatial scales may be an artifact of the Desroziers's diagnostics, and this will be assessed later through experiments. In particular, we estimate the length scale of the Gaussian correlation function to be equal to $40 \mathrm{~km}$, through nonlinear curve fitting.

The bottom panel shows only the fitted curves in form of spatial correlations (i.e., the two Gaussians and the resulting curve in red). Note that the shortscale Gaussian correlation (with 3-km length scale) goes to about zero in correspondence of the actual satellite data resolution $(7 \mathrm{~km}$, indicated by the blue vertical line in the panel). This is associated to error covariance with negligible spatial correlations, beyond the nominal resolution of satellite altimetry and supposedly due to the glider data contribution. Finally, considering Eq. (6), our assessment implies that half of the observational error is not correlated $\left(\mathbf{O}_{k}\right)$, while the other half is correlated $\left(\mathbf{N}_{k}\right)$ with a length scale of $12 \mathrm{~km}$. 

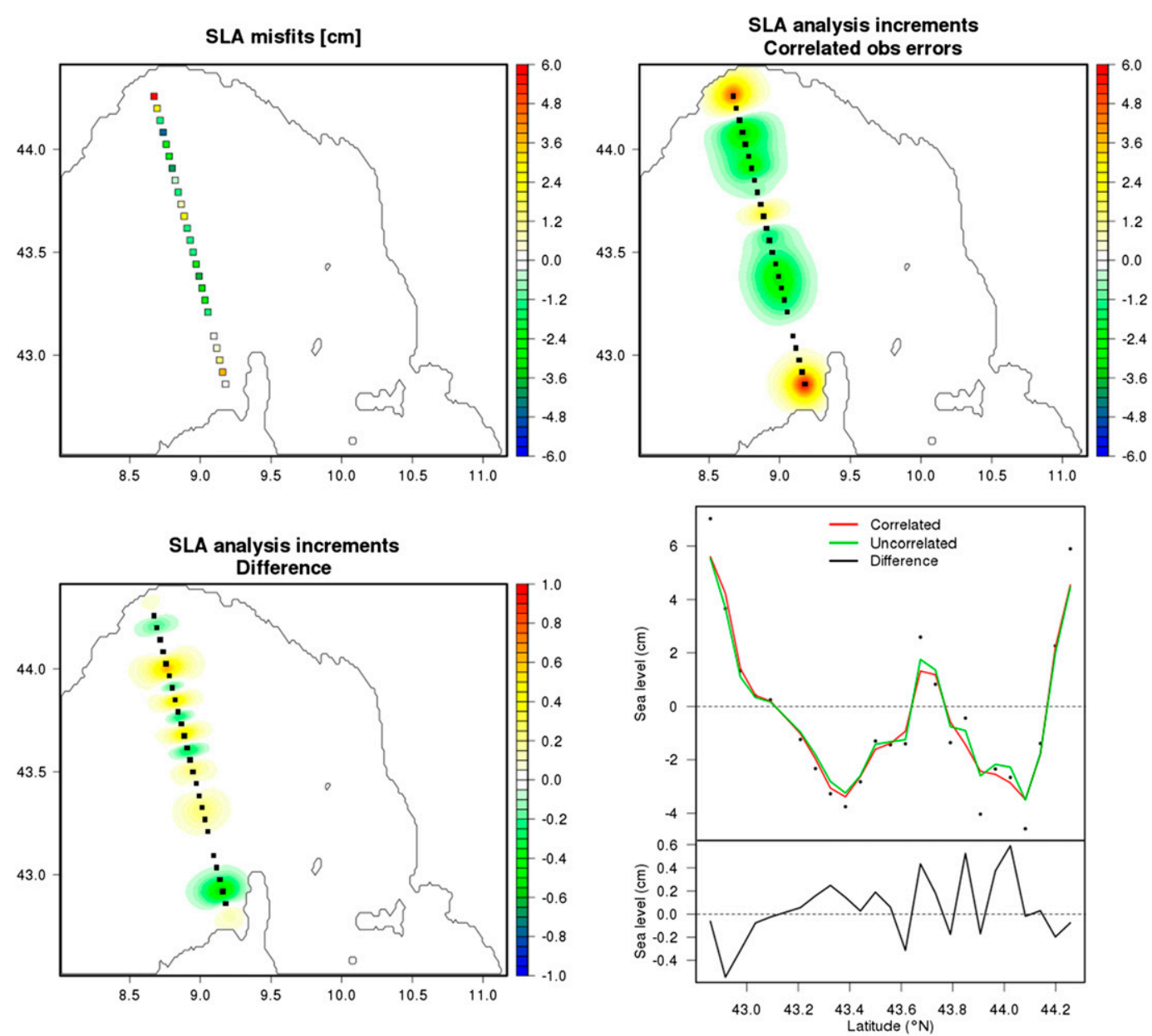

FIG. 4. Altimetry single-track assimilation experiments: (top left) the innovations (or misfits) of the SLA observations assimilated, (top right) the SSH analysis increments when along-track error correlations are imposed and (bottom left) their difference with respect to the case when along-track error correlations are neglected, and (bottom right) the misfits (black dots) and analysis increments in observation space, following the altimetry track.

\section{c. Single-track assimilation experiments}

To illustrate the effect of uncorrelated altimetry errors, several single-track assimilation experiments were performed. Figure 4 shows the SLA misfits (topleft panel; $\mathrm{cm})$ and the sea surface height analysis increments for the case of correlated altimetry observation errors and their difference with respect to the uncorrelated error case (top-right and bottom-left panels, respectively), while the bottom-right panel shows the same quantities in observation space, along the altimetry track. As expected, allowing error correlations reduces, in absolute values, the analysis increments for close observations with similar misfits (e.g., in correspondence of the negative innovations at around $44.0^{\circ}$ and $43.3^{\circ} \mathrm{N}$ ). Off-diagonal terms of $\mathbf{R}$ attenuate the impact of SLA data, assuming that their errors are correlated in space (Liu and Rabier 2003); therefore, differences between the experiments with correlated minus uncorrelated altimetry errors exhibit values of opposite sign to the analysis increments themselves. Introducing error correlations acts similarly to increasing the observational errors for the group of observations whose innovations are concordant. It is worth noting that the actual impact may also depend on the background-error correlation length scales.

\section{Impact assessments}

\section{a. Configuration of the experiments}

Based on the formulation and results of section 3, we present here the impact assessment of the correlated altimetry error formulation. For comparison, another experiment is run, using the original formulation with uncorrelated observation errors. The experiments are 
setup so that the error variance is the same regardless of the error covariance terms, namely in the case of uncorrelated altimetry errors, the only difference is that the matrix $\mathbf{C}_{k}$ in Eq. (6) is equal to the identity matrix. When the errors are not correlated, the total error variance is given by the sum of instrumental, representativeness, and MDT error variances (Storto et al. 2011). The representativeness error variance is calculated according to the Oke and Sakov (2008) method: it is the spatial variance of the anomaly within moving boxes with respect to the moving box average; we used moving boxes of size equal to $9 \times 9$ grid points. Such an error peaks at around $1 \mathrm{~cm}$ around coastal areas and has smaller values elsewhere (not shown). Instrumental errors are set equal to $2 \mathrm{~cm}$ for Jason-3 and Sentinel-3, $3 \mathrm{~cm}$ for AltiKa, and $4 \mathrm{~cm}$ for CryoSat-2. When the errors are correlated, $50 \%$ of the error covariance calculated as above is allowed to have along-track spatial correlations, based on the assessment in section $3 \mathrm{~b}$.

The experiments are indicated as Rdiag and Ratcor to identify the use of diagonal or along-track correlation for the altimetry errors, respectively. Additionally, an experiment Ratcor2 is also performed, where the error correlation length scale for altimetry data is increased to $45 \mathrm{~km}$, which comes from the Desroziers diagnostic (see section $3 b$ ), applied to the Ratcor assimilation output diagnostics. Such an experiment allows us to evaluate the impact of a longer length scale than that computed with our method, in agreement with such assimilation output diagnostics. Note that, according to Waller et al. (2016), when such diagnostics are used for length-scale calculation, then it is recommended to use assimilative experiments with correlated errors. This is the reason why the diagnostics are applied to the Ratcor experiment rather than Rdiag. Furthermore, skill score metrics also present root-mean-square error (RMSE) values from the control experiment (Ctrl).

The experiments have been run for the period from 1 August until 14 November 2017 and results are evaluated for the period from 10 October 2017 until the end of the experiments, to allow sufficient spinup of the ocean model, which is initialized on 1 August 2017 from the ocean at rest and the initial conditions of temperature and salinity extracted from the CMEMS Mediterranean Sea Forecasting system (available from the CMEMS catalogue at http://marine.copernicus.eu/ services-portfolio/access-to-products). Note that although altimetry error correlation length scales are estimated using data during the same experimental period, only 4 tracks fall in the verification period (Table 1) out of the 61 tracks assimilated. Moreover, AltiKa and CryoSat-2 sea level anomalies are assimilated using the same error correlation structure as Jason-3 and
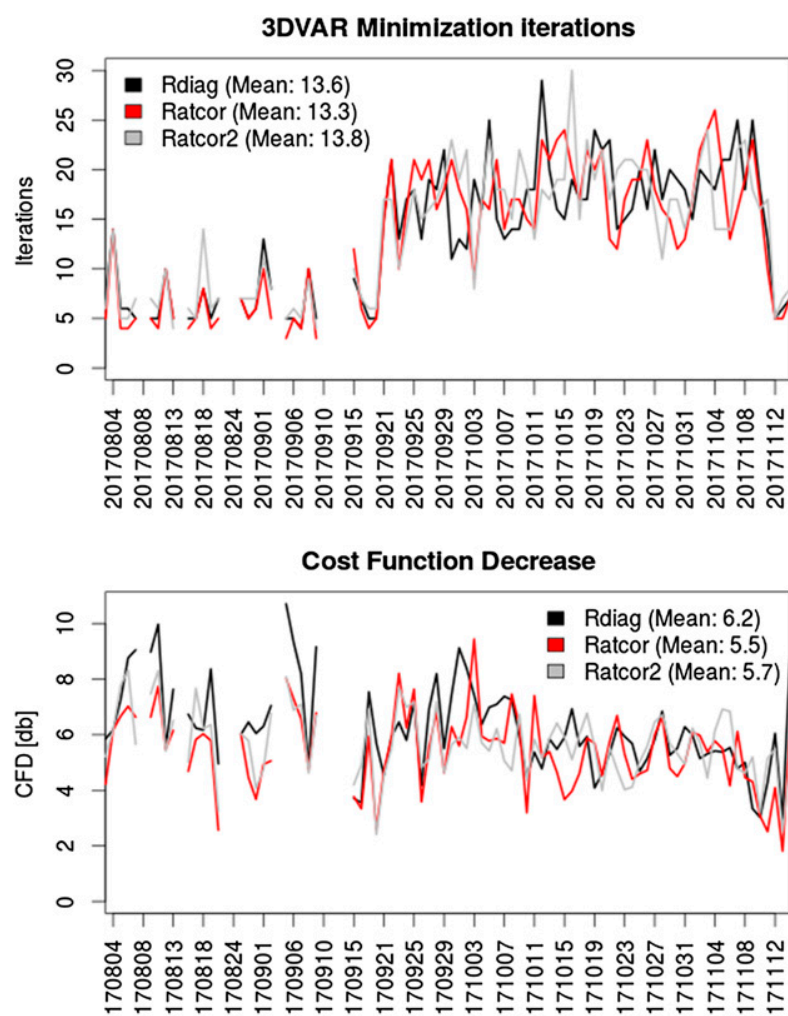

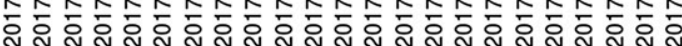

FIG. 5. (top) Number of iterations needed by the minimizer to converge for the three experiments presented in the text during the experimental period, with a fixed convergence criterion on the cost function gradient decrease. (bottom) Cost function decrease $(\mathrm{CFD} ; \mathrm{dB})$ for the three experiments. The legend reports the timemean values as well.

Sentinel-3, thus limiting the conceptual problem of data dependence, although the use of same length scales for all missions might be suboptimal.

The assimilation is performed every day using daily assimilation time window; each day a 5-day forecast is run. Note that although the experimental period is relatively short, the availability of glider data from LOGMEC17 provides a valuable verification dataset to assess the introduction of correlated altimetric observational errors.

\section{b. Impact on the cost function minimization}

First of all, we assess the performance of the analysis scheme in terms of variational minimization convergence. Because of the small number of observations assimilated, the cost function convergence is expected not to be significantly affected. Nevertheless, this is an aspect to verify, as the off-diagonal terms of $\mathbf{R}$ may, in principle, worsen the preconditioning of the minimization and slow down the convergence. Figure 5 (top panel) shows the number of iterations needed by the 
minimizer to converge, provided that the convergence criterion is the same in the two experiments: convergence is reached when the infinity norm of the cost function gradient (maximum of the absolute value of the gradient components) is smaller than $3 \%$ of the initial cost function gradient. It turns out that the number of iterations is very similar between the three experiments and equal on the average to 13.6, 13.3, and 13.8 for Rdiag, Ratcor, and Ratcor2, respectively, showing an occasional slight decrease due to the correlated observational error formulation. Note also that the sharp increase of iterations after 21 September 2017 is due to the ingestion of glider observations, opposed to the early period when in practice only altimetry data are available over the model domain.

The bottom panel of Fig. 5 shows the cost function decrease (CFD), which is defined here as $\mathrm{CFD}=10 \log _{10}\left|J_{i} / J_{f}\right|$, with $J_{i}$ and $J_{f}$ the initial and final cost function values (at the beginning and end of the minimization), that is, using a logarithmic expression to exalt the changes along the minimization. CFD is expressed in decibels $(\mathrm{dB})$, and the larger its value is, the larger is the decrease of the cost function during the variational minimization. Time-mean values for CFD are 6.2 (Rdiag), 5.5 (Ratcor), and $5.7 \mathrm{~dB}$ (Ratcor2). In particular, Ratcor exhibits the smallest decrease during the first 20 days of the experiment when only altimetry data are available, opposite to the behavior during the period from 21 September 2017 where values are close, suggesting that when only the altimetry data are present, the correlated observation error formulation may lead to smaller cost function decrease; however, this becomes negligible when glider data are available, as the cost function is dominated by the glider innovations.

\section{c. Impact on the analysis increments}

The impact on the new formulation of $\mathbf{R}$ is shown in Fig. 6 in the comparison of analysis increments of sea level and upper-ocean (top $30 \mathrm{~m}$ ) temperature. Analysis increments in Ratcor (top panels) exhibit a lowering of the sea surface height in the middle of the domain; cooling of the upper ocean is associated to such lowering. The difference of the analysis increments (middle panels) show a rise/warming in the middle of the domains corresponding to the central part of the altimetry tracks assimilated. Consistently with the single-track assimilation experiments (Fig. 4), the rise confirms that the spatial correlation attenuates the analysis increments caused by congruous altimetry innovations. The difference of analysis increments standard deviation (bottom panels) provides a diagnostics on the different variability borne from the analysis onto the forecasts. Allowing spatial correlation errors for altimetry tracks slightly damps out the spatial variability of analysis increments, especially in the central part of the domain, where the variability itself is the highest (not shown). However, the upper-ocean temperature exhibits local increase of standard deviation, suggesting that subsurface propagation of the altimetry innovation may occasionally amplify the variability of the analysis increments.

In Ratcor2 (Fig. 7), the averaged analysis increments look close to those in Ratcor, although differences with respect to Rdiag are slightly amplified in the western part of the modeling domain (middle-left panel). The variability of sea level increments (bottom-left panel) is further damped in Ratcor2, with decrease of standard deviation reaching locally $1 \mathrm{~cm}$ in the western area of the domain. Conversely, the longer correlation length-scale impacts only marginally the temperature increments, in terms of both mean and standard deviation of differences compared to Rdiag. Differences of salinity analysis increments between the three experiments are small (generally less than $0.02 \mathrm{psu}$; not shown).

\section{d. Impact on skill score verification metrics}

Figure 8 shows vertical profiles of bias and standard deviation of innovations, calculated against in situ data during the first and fifth days of forecast. Verification skill scores are calculated against glider data collected in proximity of the altimetry tracks, without any processing or quality control of them. We show bias and standard deviation of innovations in order to decompose the RMSE into systematic (bias, observation minus forecast fields) and random (standard deviation) errors, considering that $\mathrm{RMSE}^{2} \cong \operatorname{bias}^{2}+\operatorname{std~dev}^{2}$. Temperature biases are warm within the mixed layer. Ratcor exhibits significantly smaller biases in the top $50 \mathrm{~m}$ after both 1 and 5 days of forecasts. Between depths of 150 and $300 \mathrm{~m}$, Ratcor shows a small warm bias while Rdiag a small cold bias, Ratcor's one being slightly smaller in absolute values. After five days of forecasts, the differences are not distinguishable. Below a depth of $300 \mathrm{~m}$, differences amplify with the forecast lead and after 5 days Ratcor shows a smaller cold bias than Rdiag. Ratcor2 has a bias profile close to Ratcor, although nearsurface (top $30 \mathrm{~m}$ ) and deep ocean (below $300 \mathrm{~m}$ ) biases are amplified. However, the variability (standard deviation, top-right panel) of temperature innovations is not significantly different between the two experiments, indicating that the introduction of along-track correlations acts mostly on the mean fields rather than their variability. Ratcor 2 performs worse than other experiments in the top $10 \mathrm{~m}$, and slightly better in correspondence of the mixed layer depth (maximum of the error). Note that the standard deviation slightly reduces with forecast time in the top $10-20 \mathrm{~m}$, likely because of the 
Mean Sea Level Analysis increments [mm] Ratcor
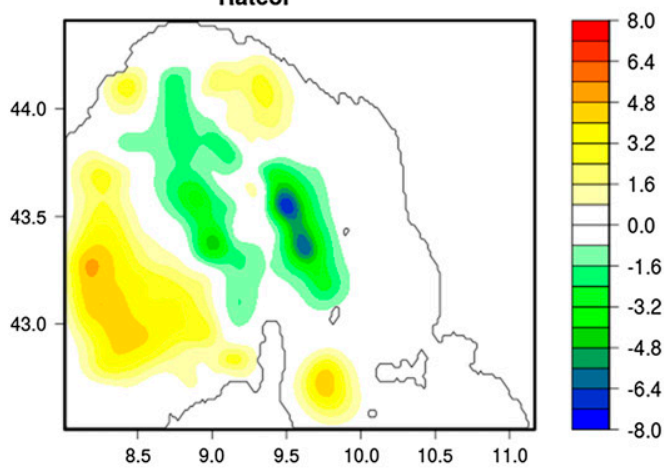

$-1.6$

$-3.2$

$-4.8$

$-6.4$

$-8.0$

Mean Sea Level Analysis increments [mm] Ratcor - Rdiag

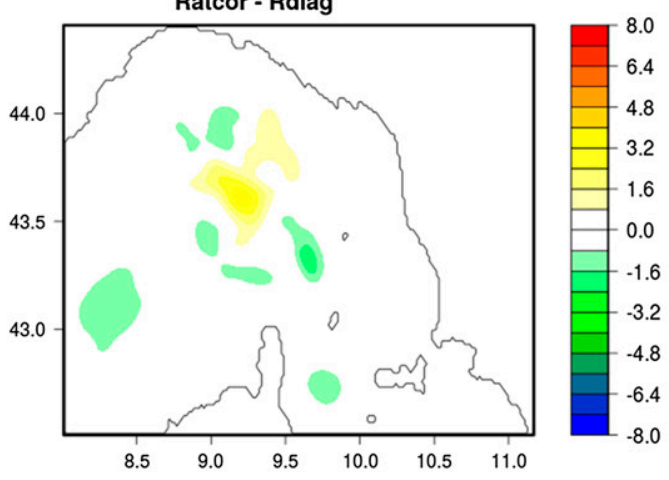

St. Dev. Sea Level Analysis increments [mm]

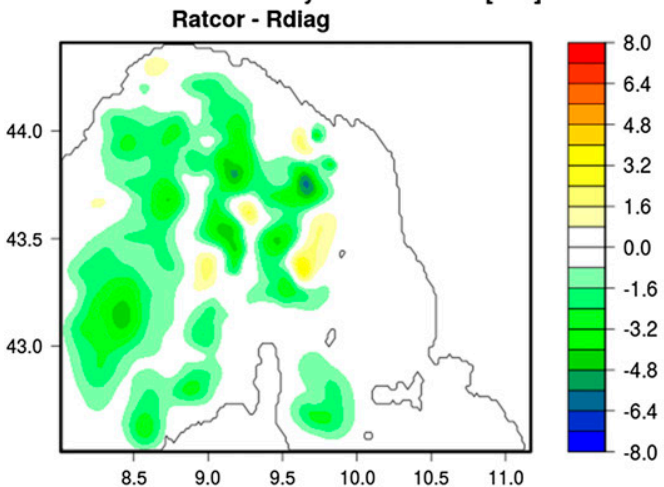

Mean Temperature Analysis increments $\left[{ }^{\circ} \mathrm{C}\right]$ Ratcor [0-30m]

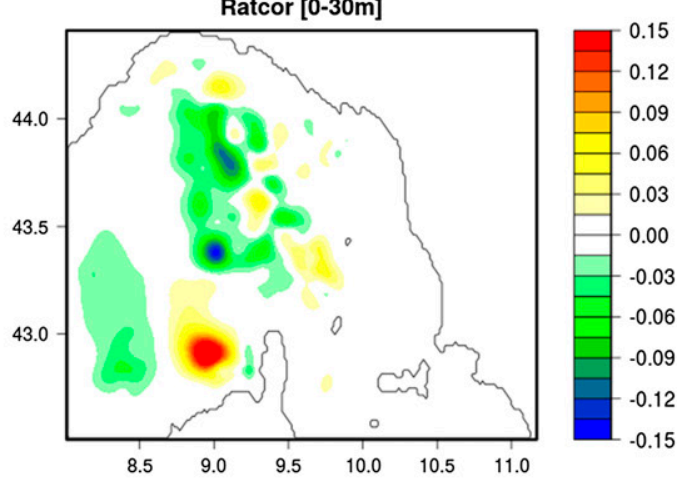

Mean Temperature Analysis increments $\left[{ }^{\circ} \mathrm{C}\right]$ Ratcor - Rdiag [0-30m]

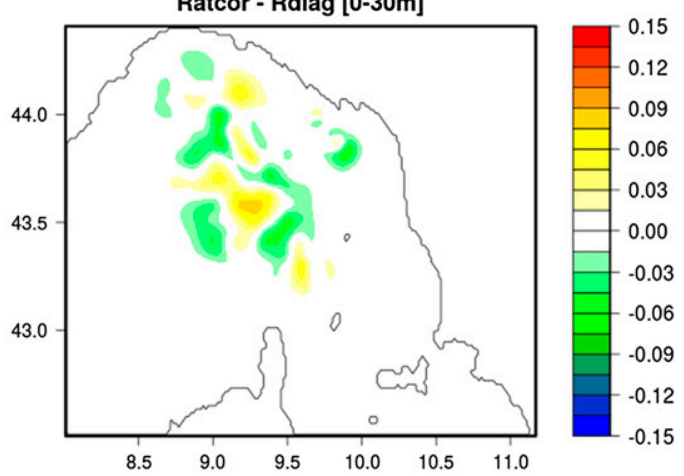

St. Dev. Temperature Analysis increments $\left[{ }^{\circ} \mathrm{C}\right]$

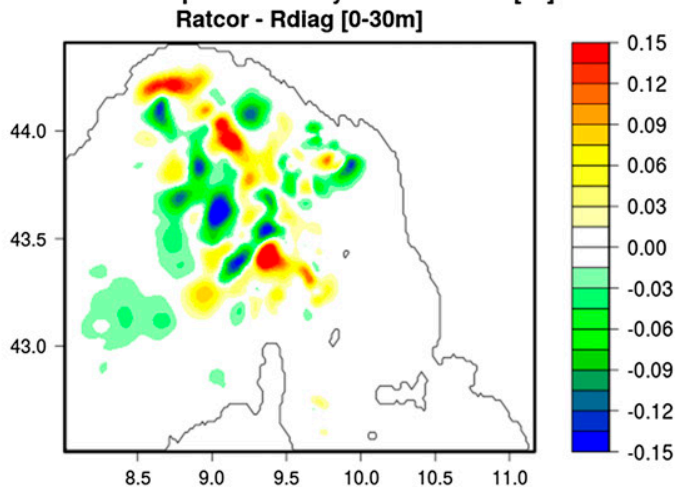

FIG. 6. (top) Time-averaged analysis increments in Ratcor, (middle) time-averaged differences of analysis increments between Ratcor and Rdiag, and (bottom) the difference of analysis increment standard deviations. The diagnostics are shown for (left) sea level and (right) upper-ocean (top $30 \mathrm{~m}$ ) temperature.

effect of SST nudging in the verification against glider data at the very beginning of the forecast step, while it increases with time in correspondence of the mixed layer depth.

Salinity profiles of bias show a more irregular structure and with slighter differences between the experiments than the temperature. Rdiag shows a smaller bias (in absolute values) than Ratcor in the top $80 \mathrm{~m}$ and between depths of 150 and $250 \mathrm{~m}$, while larger elsewhere, after 1 day of forecast. After 5 days of forecast, bias profiles are very similar between the two experiments in the upper ocean (top $150 \mathrm{~m}$ ), but below $150 \mathrm{~m}$, Ratcor shows smaller biases in absolute values. Biases below a depth of $100 \mathrm{~m}$ significantly increase along the 5 days of forecasts. Ratcor 2 performs similarly to Ratcor, except below a depth of $350 \mathrm{~m}$ where it exhibits 

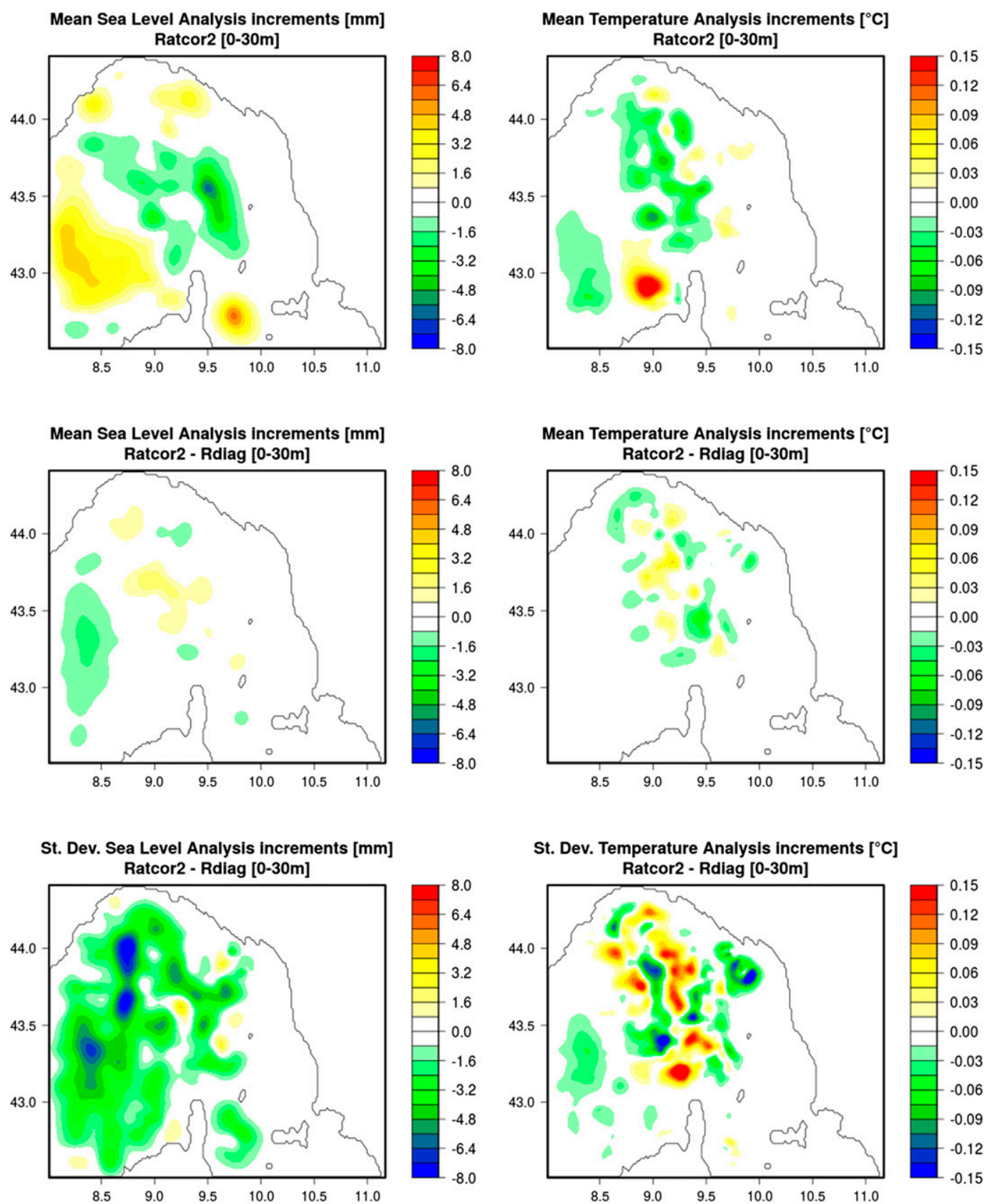

FIG. 7. As in Fig. 6, but for Ratcor2.

large fresh biases. The variability seems less affected, like for temperature, with the use of along-track error correlations leading to slightly worse profiles after 1 day and below a depth of $100 \mathrm{~m}$, while smaller standard deviations after 5 days of forecasts. Also the variability of Ratcor2 shows the detrimental impact of long correlation length scales below a depth of $300 \mathrm{~m}$.

The assessment of the experiments in terms of RMSE as a function of forecast day is presented in Figs. 9-11 for the validation of the forecasts against in situ profiles (glider data), sea surface temperature, and sea surface height. In situ profiles and altimetry data are assimilated in the system; nevertheless, they represent an independent forecast validating datasets if one neglects the temporal correlation of the observation errors. For in situ data validation, skill scores are computed separately for four vertical regions (depths of 0-30, 30-100, 100300 , and $300-800 \mathrm{~m}$ ). SST observations come from the nighttime measurements of SST swath data (L3 product) from AVHRR sensors onboard several satellites 
Temperature Innovation Bias

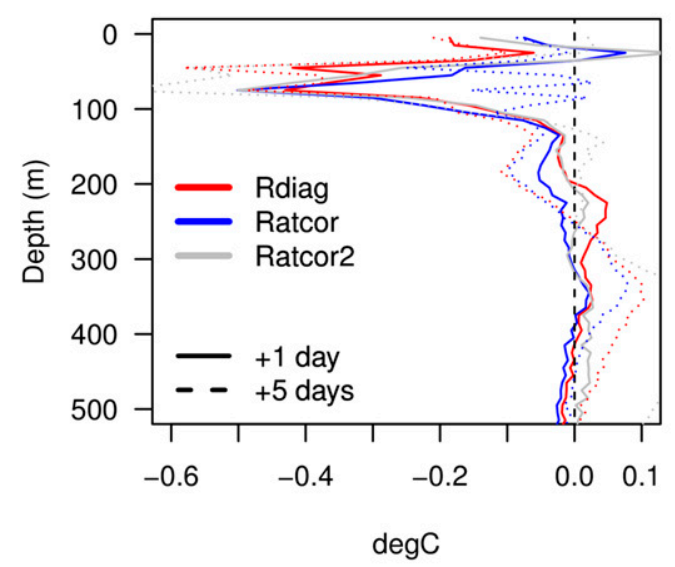

\section{Salinity Innovation Bias}

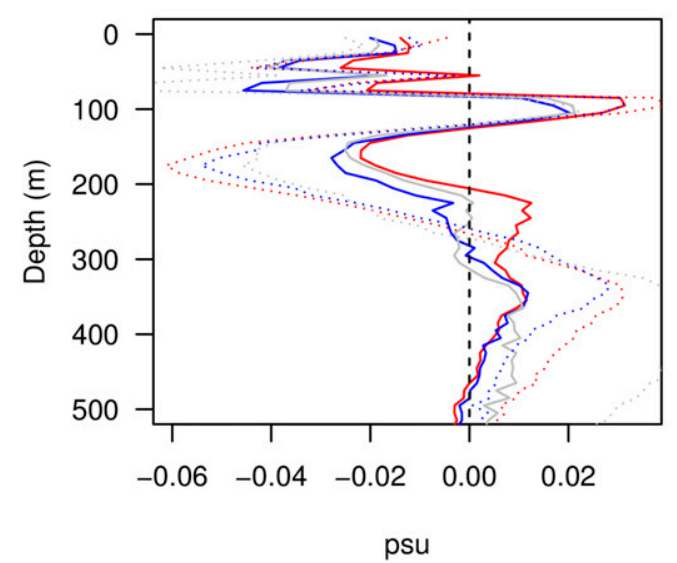

Temperature Innovation Standard deviation

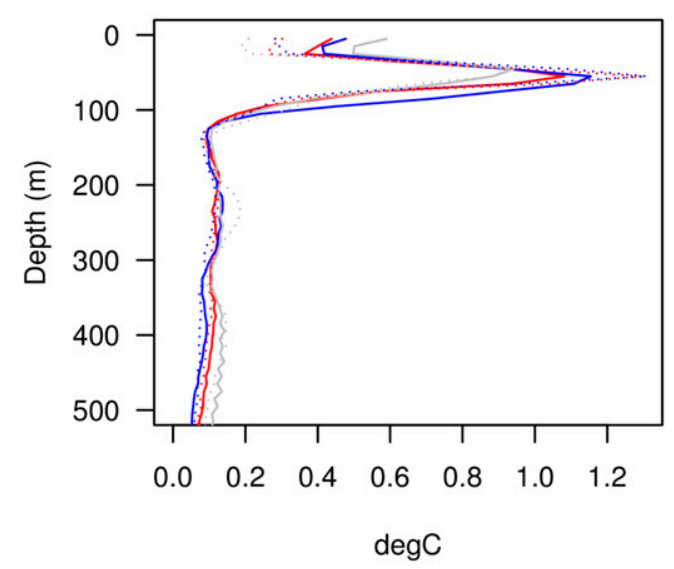

Salinity Innovation Standard deviation

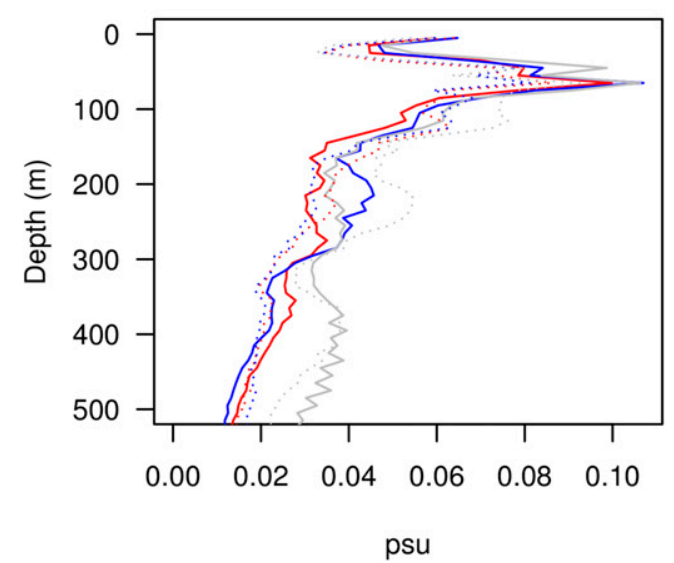

FIG. 8. Vertical profiles of (left) bias and (right) standard deviation of innovations (misfits) calculated from the glider profiles in proximity of the altimetry tracks for (top) temperature and (bottom) salinity. Solid (dotted) lines show the scores during the first (fifth) day of the forecast.

(MetOp-B, MODIS, Suomi NPP) and processed by CMEMS (Buongiorno Nardelli et al. 2015). Only nighttime measurements are considered, in order not to compromise the scores with possible misrepresentation of the diurnal cycle by the ocean model with respect to the satellite skin SST. RMSE percentage differences (reported as text in the figures) are significant when larger than or smaller than about $1.5 \%-1.8 \%$ (depending on the different number of validating observations), based on the Welch's $t$ test at 95\% confidence level and performed on the squared innovations.

The upper-ocean (top $30 \mathrm{~m}$ ) temperature skill scores show an impact of the along-track correlations in Ratcor growing with forecast day (except for day 5) and significantly positive at any time. Except for day 5, Ratcor2 provides detrimental results than Ratcor and, at days 1 and 2, worse than Rdiag. Similarly, score differences between depths of 30 and $100 \mathrm{~m}$ are significant (positive) and grow with forecast lead, likely because of the dominant effect of the misplacement of the mixed layer depth by the model that amplifies the impact. At forecast days $3-5$, Ratcor is again the best performing experiment. This applies also to the RMSE in the vertical regions of $100-300$ and $300-800 \mathrm{~m}$, where a significant improvement occurs (after the first day of forecast for the 100-300-m layer). In both vertical regions, the long correlation length scales estimated through the Desroziers's method (Ratcor2) provide largely negative impact, likely because of the damping of small-scale features that might better respond to the near-surface dynamics directly constrained by the atmospheric forcing. In some cases at depth (e.g., in the 300-800-m layer), 
Temperature RMSE (0-30m) [degC]

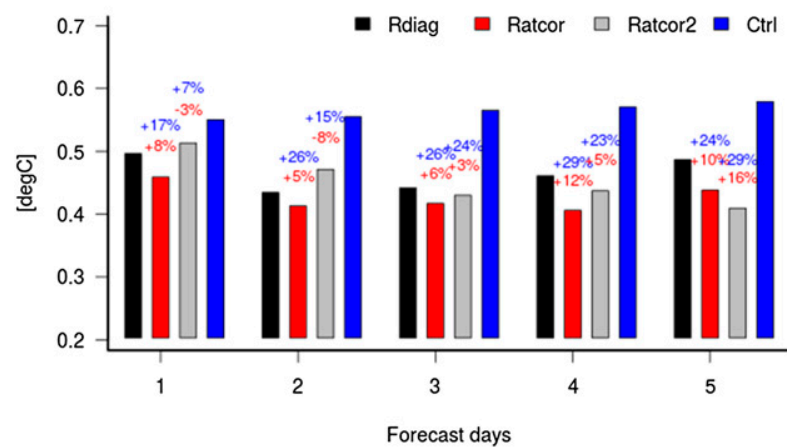

Temperature RMSE (100-300m) [degC]

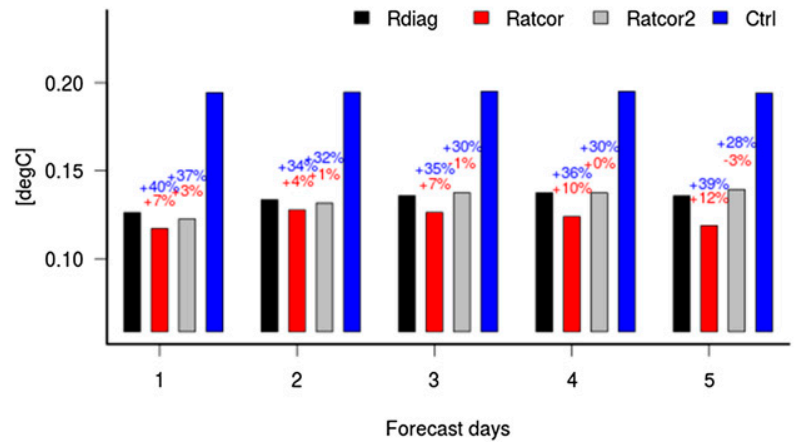

Temperature RMSE $(30-100 \mathrm{~m})$ [degC]

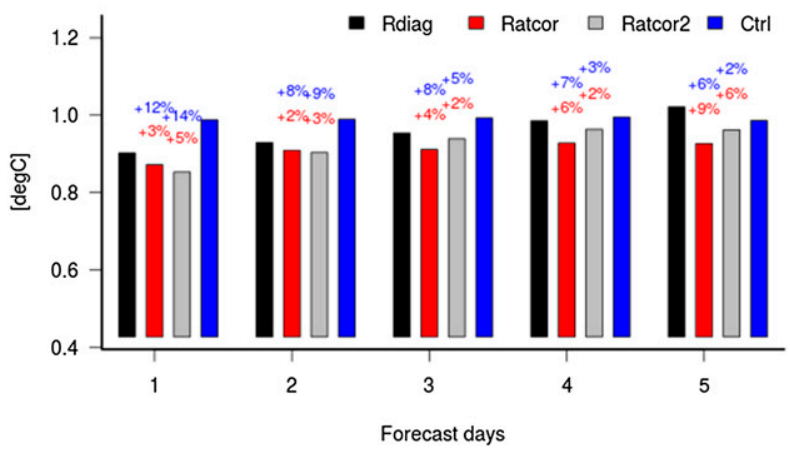

Temperature RMSE $(300-800 \mathrm{~m})$ [degC]

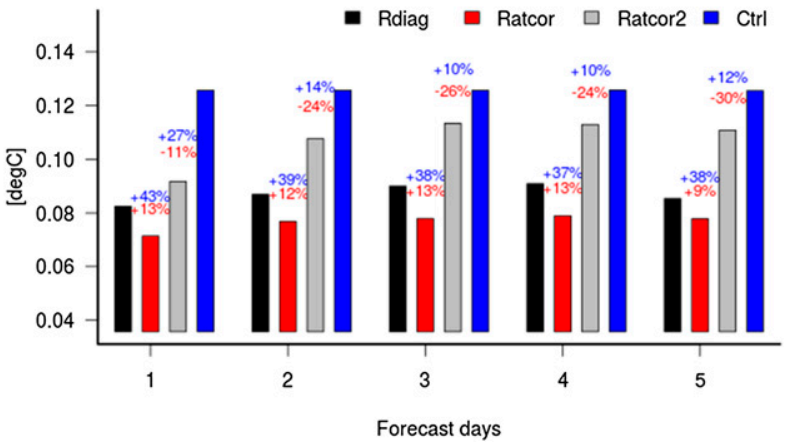

FIG. 9. RMSE as a function of forecast day in the verification against glider data of temperature for four different vertical regions and the experiments presented in the text. Percentages above the bars refer to the RMSE decrease of Ratcor and Ratcor2 with respect to Rdiag and Ctrl in red and blue, respectively. Positive values indicate decrease of RMSE.

RMSE values do not always increase monotonically with the forecast range, because of the limited and largely irregular sampling of the verifying glider data. This feature is indeed present also in the verification of control experiments, or with the use of other atmospheric forcing, or in the verification against persistence (not shown), indicating that does not depend on possible initialization shocks but only on the sampling of the verifying glider data, to which no preprocessing nor quality control is performed for verification purposes.

RMSE results for salinity show neutral results, compared to the significance threshold, for the vertical regions of $0-30$ and $30-100 \mathrm{~m}$, although generally Rdiag performs slightly better than others, in agreement with the bias profile of Fig. 8. Ratcor exhibits largely positive results in the range of 100-300 m (significant RMSE decrease after the first forecast day), while positive impact in the depth range of 300-800 m occurs up to day 4 , and turns to neutral at day 5. Conversely, Ratcor2 performs worse than Ratcor in the 100-300-m layer and even worse than Rdiag in the 300-800-m layer.

Skill score statistics in the verification against SST and SLA observations (Fig. 11) suggest that there exist small but positive impact in all the forecast ranges in Ratcor.
However, the impact on SLA verification is significant (at day 5), while that on SST is on the order of $1 \%$. Ratcor2 provides a detrimental impact compared to Rdiag in the SST verification, while neutral in the verification against SLA. Note also that for all skill scores there exist a significant and large improvement from the assimilation compared to the Ctrl experiment, although small in the verification against SST data at day 1 . This can be ascribed to the suboptimal assimilation scheme for SST, based on the air-sea heat flux correction through nudging, and possibly the inconsistency between the assimilated data (L4 SST product) and the verifying data (nighttime multisensor L3 data). The SST RMSE decrease with respect to Ctrl appears however significant after day 2, indicating that the forecast uncertainty exceeds the suboptimality of the SST assimilation scheme since then.

\section{Summary and discussion}

In this article, we have investigated the feasibility of relaxing the assumption of uncorrelated altimetry observational error in a variational analysis scheme, in order to improve the assimilation of sea level anomaly data. The collocations between altimetry data and glider 

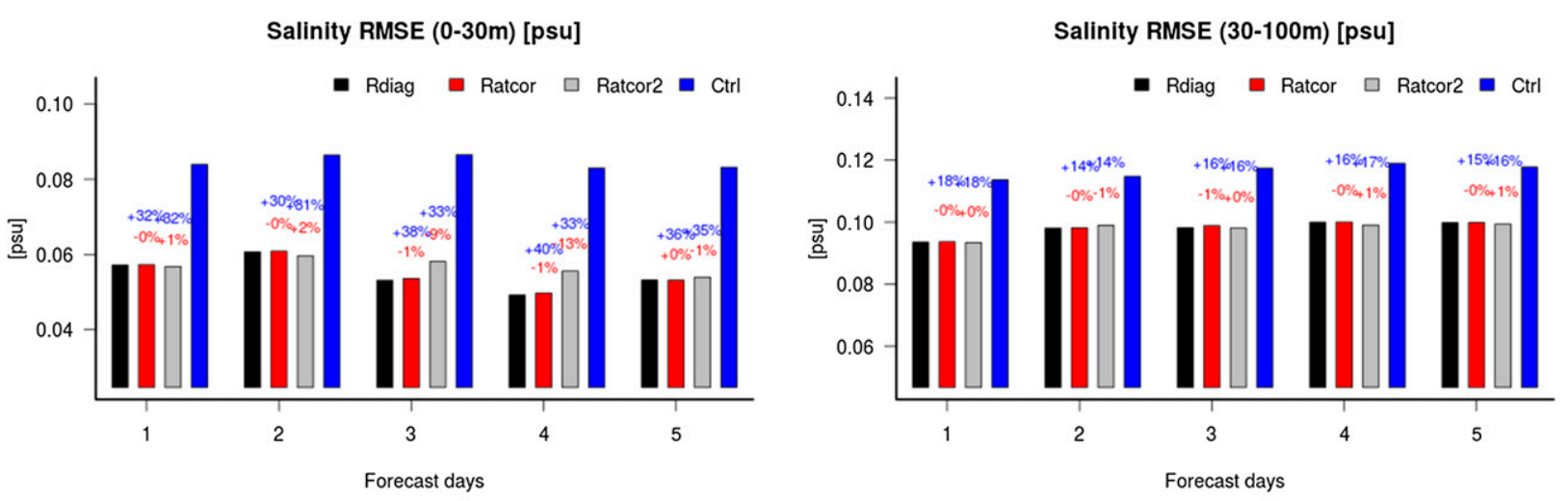

Salinity RMSE (100-300m) [psu]
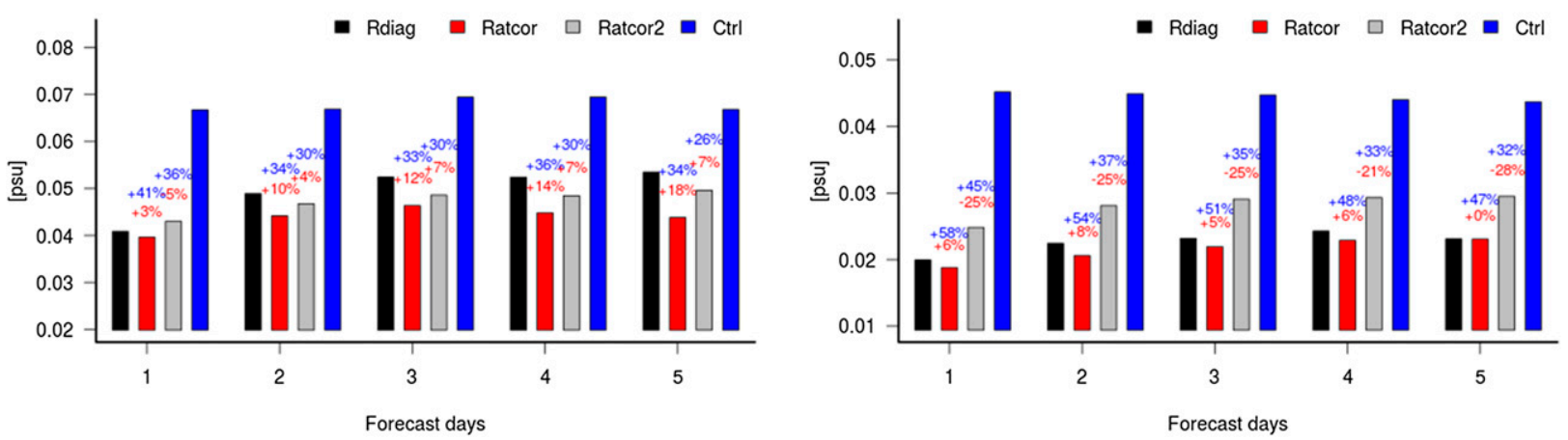

FIG. 10. As in Fig. 9, but for the verification against glider data of salinity.

profiles of temperature and salinity provide a dataset to infer the error correlation length scales of altimetry data, assuming that the horizontal error correlation of the integrated glider data is negligible or at least shorter than the resolution of altimetry data. While this assumption might not necessarily be verified in reality, it is not possible to decompose the spatial correlation length scales without an independent dataset. However, the sampling of glider data mostly concerns the smallscale oceanic structures, while altimetry data are known to be heavily processed and filtered, in a way that it is reasonable to assume that their spatial correlation is dominant. The approach used here is inspired by the Hollingsworth and Lönnberg (1986) innovation method and is generally applicable to any set of collocated observations with independent errors, although it is rare to have in the ocean different observing networks measuring the same ocean parameter, especially in limitedarea models. Furthermore, comparing the Desroziers's method (Desroziers et al. 2005) applied to the SLA assimilation outputs with our estimates confirms that the SLAs are characterized by nonnegligible spatial
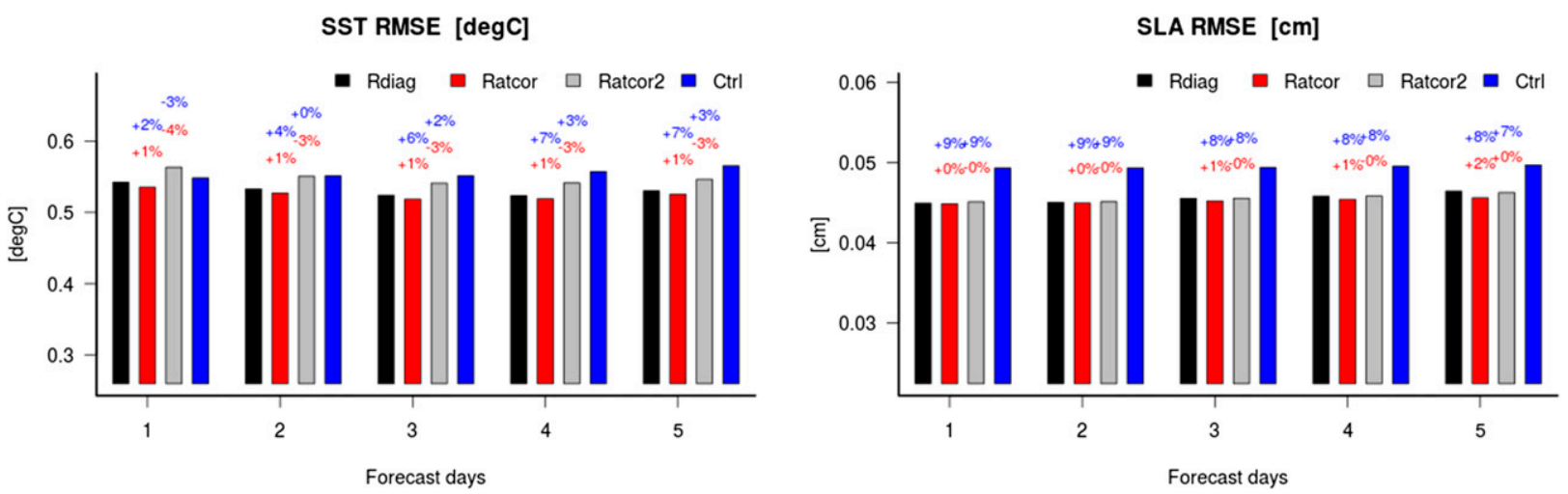

FIG. 11. As in Fig. 9, but for the verification against satellite data of (left) sea surface temperature and (right) sea level anomaly. 
correlations of the error. Discrepancy between the two methods (with Desroziers's estimates longer by about 3 times) may be ascribed to the fact that the Desroziers's method may provide incorrect estimates when, for example, errors in the system are not well specified or observation and background error length scales are comparable (Desroziers et al. 2010; Waller et al. 2016; Michel 2018). Furthermore, error correlations in unfiltered real-time along-track sea level anomalies may arise from several factors, including the small wavelength altimetry retracking noise (Dibarboure et al. 2014), the high-resolution operational atmospheric analyses used within the dynamical atmospheric corrections (e.g., Ruggiero et al. 2016), the barotropic model used within the tidal correction (Stammer et al. 2014), and the procedure to ingest in situ and drifter data within the computation of the mean dynamic topography (Rio et al. 2014). It is not an easy task to identify which are, among the latter, the factors introducing spatially correlated errors at most, because of the lack of anchoring data for each of the processing step; for instance, assessing the error length scales of atmospheric analyses is challenging and generally performed with either the Hollingsworth and Lönnberg (1986) method or through ensemble anomaly evaluation. Nevertheless, from a data assimilation perspective, it is enough to estimate the resulting error length scale of the assimilated dataset.

The results of the collocation-based assessment suggest that altimetry data are characterized by along-track correlation length scales equal to about $12 \mathrm{~km}$, for the CMEMS dataset used and in the midlatitude region of study. Given the small area of study, we consider this length scale homogeneous spatially and among satellites, but this could change for large domains. For instance, the atmospheric data used for the atmospheric corrections of the radar altimetry signal may induce inhomogeneous correlations length scales. For coarse resolution or global configurations this length scale is generally comparable or smaller than the typical resolution of ocean models, and usual subsampling ("thinning" or "super-obbing" procedures) used therein make the inclusion of this error spatial scale unimportant. However, for high-resolution limited area models where satellite data may be assimilated at the full resolution $(7 \mathrm{~km})$, this poses the problem of considering such error scales.

Introducing along-track altimetry error correlation was accomplished through modification of the variational cost function to include an off-diagonal error component in the definition of the observational error covariance matrix, which is usually neglected in most data assimilation systems. We argue that this approach is superior to both data thinning and observation error inflation, as it can retain all small-scale features, together with possibly large gradients in the observations (Fowler et al. 2018).

The preconditioning of the minimization is not much affected in our results, as we allow off-diagonal terms only within altimetry tracks and not for the entire observation error covariance matrix, which would require in turn more sophisticated strategies, as those suggested, for example, by Ruggiero et al. (2016) for future highresolution altimetry data from SWOT, relying, for example, on gradient assimilation. We showed indeed that for correlation scales on the order of $10-15 \mathrm{~km}$ and data resolution of less than $10 \mathrm{~km}$, the approach is in practice similar to the use of Markov matrix or tridiagonal matrices formulated by Stewart et al. (2013). Furthermore, with the preconditioning based on control variable transformation, it is acknowledged that the condition number largely depends on background-error covariances when the number of observations assimilated is much smaller than the size of the control vector, as generally occurs in regional prediction systems.

In an experimental framework that mimics a regional variational ocean prediction system with 5-day forecasts produced, our strategy to assimilate error correlationaware altimetry data proves effective in significantly reducing the forecast RMSE with respect to the reference experiment with uncorrelated altimetry errors, acting especially on the reduction of systematic errors of temperature, salinity below a depth of $100 \mathrm{~m}$, and sea surface height. The impact is found to be nonsignificant in the verification against upper-ocean (top $100 \mathrm{~m}$ ) salinity data and sea surface temperature measurements from infrared satellites. The RMSE improvement is obtained at no extra computational costs, since the new formulation does not impact the number of iterations required by the minimizer to converge. Using the long error length scale $(45 \mathrm{~km})$ from the Desroziers's method generally worsens the skill scores, especially at depth (below $300 \mathrm{~m}$ ), where such error scales may damp out the small-scale features of the unfiltered altimetry data in regions where the effect of the large-scale atmospheric forcing error is less important.

In general, we have shown that campaigns through which remote sensing data and in situ profiles are collocated represent an extremely informative tool for data assimilation systems, through which fine tuning and indepth assessment of the analysis system can be achieved. Indeed, further to using the collocated data to assess the correlation length scales, the glider tracks have offered the possibility to validate the data assimilation modifications in a robust way. Similar exercises may be repeated in different study areas or for different sensors, for example, in order to improve the assimilation of 
daytime sea surface temperature from satellites, or sea level from current and forthcoming missions.

Acknowledgments. This work is supported by the SAC000807 project "Sensing and predicting underwater noise using robotic platforms and forecast models for Maritime ISR (EKOE2/MISR2)" funded by the NATO Allied Command Transformation (ACT). The authors are grateful to Isabelle Pujol [Collecte Localisation Satellites (CLS)] for the fruitful discussion on the altimeter footprint geometry and short-wavelength correlated errors. Three anonymous reviewers helped improve the quality of the manuscript.

\section{REFERENCES}

Backeberg, B. C., F. Counillon, J. A. Johannessen, and M.-I. Pujol, 2014: Assimilating along-track SLA data using the EnOI in an eddy resolving model of the Agulhas system. Ocean Dyn., 64, 1121-1136, https://doi.org/10.1007/s10236-014-0717-6.

Barnier, B., and Coauthors, 2006: Impact of partial steps and momentum advection schemes in a global ocean circulation model at eddy-permitting resolution. Ocean Dyn., 56, 543567, https://doi.org/10.1007/s10236-006-0082-1.

Bormann, N., and P. Bauer, 2010: Estimates of spatial and interchannel observation-error characteristics for current sounder radiances for numerical weather prediction. I: Methods and application to ATOVS data. Quart. J. Roy. Meteor. Soc., 136, 1036-1050, https://doi.org/10.1002/qj.616.

Borrione, I., P. Oddo, A. Russo, and E. Coelho, 2019: Understanding altimetry signals in the northeastern Ligurian Sea using a multi-platform approach. Deep-Sea Res. I, 145, 83-96, https://doi.org/10.1016/j.dsr.2019.02.003.

Brankart, J., C. Ubelmann, C. Testut, E. Cosme, P. Brasseur, and J. Verron, 2009: Efficient parameterization of the observation error covariance matrix for square root or ensemble Kalman filters: Application to ocean altimetry. Mon. Wea. Rev., 137, 1908-1927, https://doi.org/10.1175/2008MWR2693.1.

_ - E. Cosme, C. Testut, P. Brasseur, and J. Verron, 2010: Efficient adaptive error parameterizations for square root or ensemble Kalman filters: Application to the control of ocean mesoscale signals. Mon. Wea. Rev., 138, 932-950, https://doi.org/ 10.1175/2009MWR3085.1.

Buongiorno Nardelli, B., C. Tronconi, A. Pisano, and R. Santoleri, 2013: High and ultra-high resolution processing of satellite sea surface temperature data over southern European seas in the framework of MyOcean project. Remote Sens. Environ., 129, 1-16, https://doi.org/10.1016/j.rse.2012.10.012.

_, A. Pisano, C. Tronconi, and R. Santoleri, 2015: Evaluation of different covariance models for the operational interpolation of high resolution satellite sea surface temperature data over the Mediterranean Sea. Remote Sens. Environ., 164, 334-343, https://doi.org/10.1016/j.rse.2015.04.025.

Byrne, D. A., and J. L. McClean, 2008: Sea level anomaly signals in the Agulhas Current region. Geophys. Res. Lett., 35, L13601, https://doi.org/10.1029/2008GL034087.

Carrere, L., Y. Faugère, and M. Ablain, 2016: Major improvement of altimetry sea level estimations using pressure-derived corrections based on ERA-Interim atmospheric reanalysis. Ocean Sci., 12, 825-842, https://doi.org/10.5194/os-12-825-2016.
Chelton, D. B., E. J. Walsh, and J. L. MacArthur, 1989: Pulse compression and sea level tracking in satellite altimetry. J. Atmos. Oceanic Technol., 6, 407-438, https://doi.org/10.1175/ 1520-0426(1989)006<0407:PCASLT >2.0.CO;2.

J. C. Ries, B. J. Haines, L. L. Fu, and P. S. Callahan, 2001: Satellite altimetry. Satellite Altimetry and Earth Sciences: A Handbook of Techniques and Applications, L. L. Fu and A. Cazenave, Eds., Academic Press, 1-132.

Cheney, R. E., 2001: Satellite altimetry. Encyclopedia of Ocean Sciences, 2nd ed., J. H. Steele, Ed., Academic Press, 58-64, https://doi.org/10.1016/B978-012374473-9.00340-4.

Cooper, M., and K. Haines, 1996: Altimetric assimilation with water property conservation. J. Geophys. Res., 101, 1059-1077, https://doi.org/10.1029/95JC02902.

Desroziers, G., L. Berre, B. Chapnik, and P. Poli, 2005: Diagnosis of observation, background and analysis-error statistics in observation space. Quart. J. Roy. Meteor. Soc., 131, 3385-3396, https://doi.org/10.1256/qj.05.108.

— — — , and — , 2010: Objective validation of data assimilation systems: Diagnosing suboptimality. Seminar on $\mathrm{Di}$ agnosis of Forecasting and Data Assimilation Systems, Reading, United Kingdom, ECMWF, https://www.ecmwf.int/ node/9017.

Dibarboure, G., F. Boy, J. D. Desjonqueres, S. Labroue, Y. Lasne, N. Picot, J. C. Poisson, and P. Thibaut, 2014: Investigating short-wavelength correlated errors on low-resolution mode altimetry. J. Atmos. Oceanic Technol., 31, 1337-1362, https:// doi.org/10.1175/JTECH-D-13-00081.1.

Dobricic, S., and N. Pinardi, 2008: An oceanographic threedimensional assimilation scheme. Ocean Modell., 22, 89-105, https://doi.org/10.1016/j.ocemod.2008.01.004.

Dufau, C., M. Orsztynowicz, G. Dibarboure, R. Morrow, and P.-Y. Le Traon, 2016: Mesoscale resolution capability of altimetry: Present and future. J. Geophys. Res. Oceans, 121, 4910-4927, https://doi.org/10.1002/2015JC010904.

El Akkraoui, A., Y. Trémolet, and R. Todling, 2013: Preconditioning of variational data assimilation and the use of a bi-conjugate gradient method. Quart. J. Roy. Meteor. Soc., 139, 731-741, https://doi.org/10.1002/qj.1997.

Fowler, A. M., S. L. Dance, and J. A. Waller, 2018: On the interaction of observation and prior error correlations in data assimilation. Quart. J. Roy. Meteor. Soc., 144, 48-62, https:// doi.org/10.1002/qj.3183.

Fu, L.-L., D. B. Chelton, P.-Y. Le Traon, and R. Morrow, 2010: Eddy dynamics from satellite altimetry. Oceanography, 23, 14-25, https://doi.org/10.5670/oceanog.2010.02.

Gaultier, L., C. Ubelmann, and L. Fu, 2016: The challenge of using future SWOT data for oceanic field reconstruction. J. Atmos. Oceanic Technol., 33, 119-126, https://doi.org/10.1175/JTECHD-15-0160.1.

Golub, G., and C. F. Van Loan, 2012. Matrix Computations. 4th ed. Johns Hopkins University Press, 756 pp.

Haben, S. A., A. S. Lawless, and N. K. Nichols, 2011: Conditioning and preconditioning of the variational data assimilation problem. Comput. Fluids, 46, 252-256, https://doi.org/10.1016/ j.compfluid.2010.11.025.

Hollingsworth, A., and P. Lönnberg, 1986: The statistical structure of short-range forecast errors as determined from radiosonde data. Part I: The wind field. Tellus, 38A, 111-136, https:// doi.org/10.1111/j.1600-0870.1986.tb00460.x.

Le Traon, P.-Y., and Coauthors, 2017: The Copernicus Marine Environmental Monitoring Service: Main scientific achievements and future prospects. Mercator Ocean J., 56, 1-101. 
Liu, Z. Q., and F. Rabier, 2003: The potential of high-density observations for numerical weather prediction: A study with simulated observations. Quart. J. Roy. Meteor. Soc., 129, 3013-3035, https://doi.org/10.1256/qj.02.170.

Madec, G., and Coauthors, 2012: NEMO ocean engine. Note du Pole de Modélisation de l'Institut Pierre-Simon Laplace 27, $217 \mathrm{pp}$.

Michel, Y., 2018: Revisiting Fisher's approach to the handling of horizontal spatial correlations of observation errors in a variational framework. Quart. J. Roy. Meteor. Soc., 144, 20112025, https://doi.org/10.1002/qj.3249.

Morrow, R., A. Carret, F. Birol, F. Nino, G. Valladeau, F. Boy, C. Bachelier, and B. Zakardjian, 2017: Observability of finescale ocean dynamics in the northwestern Mediterranean Sea. Ocean Sci., 13, 13-29, https://doi.org/10.5194/os-13-13-2017.

Oddo, P., A. Bonaduce, N. Pinardi, and A. Guarnieri, 2014: Sensitivity of the Mediterranean sea level to atmospheric pressure and free surface elevation numerical formulation in NEMO. Geosci. Model Dev., 7, 3001-3015, https://doi.org/10.5194/ gmd-7-3001-2014.

- A. Storto, S. Dobricic, A. Russo, C. Lewis, R. Onken, and E. Coelho, 2016: A hybrid variational-ensemble data assimilation scheme with systematic error correction for limited-area ocean models. Ocean Sci., 12, 1137-1153, https://doi.org/ 10.5194/os-12-1137-2016.

Oke, P. R., and P. Sakov, 2008: Representation error of oceanic observations for data assimilation. J. Atmos. Oceanic Technol., 25, 1004-1017, https://doi.org/10.1175/2007JTECHO558.1.

—, G. B. Brassington, D. A. Griffin, and A. Schiller, 2008: The Bluelink Ocean Data Assimilation System (BODAS). Ocean Modell., 21, 46-70, https://doi.org/10.1016/j.ocemod.2007.11.002.

Pettenuzzo, D., W. G. Large, and N. Pinardi, 2010: On the corrections of ERA-40 surface flux products consistent with the Mediterranean heat and water budgets and the connection between basin surface total heat flux and NAO. J. Geophys. Res., 115, C06022, https://doi.org/10.1029/2009JC005631.

Pujol, M.-I., S. Dobricic, N. Pinardi, and M. Adani, 2010: Impact of multialtimeter sea level assimilation in the Mediterranean forecasting model. J. Atmos. Oceanic Technol., 27, 2065-2082, https://doi.org/10.1175/2010JTECHO715.1.

— , Y. Faugère, G. Taburet, S. Dupuy, C. Pelloquin, M. Ablain, and N. Picot, 2016: DUACS DT2014: The new multi-mission altimeter data set reprocessed over 20 years. Ocean Sci., 12, 1067-1090, https://doi.org/10.5194/os-12-1067-2016.

Quartly, G. D., 1998: Determination of oceanic rain rate and rain cell structure from altimeter waveform data. Part I: Theory. J. Atmos. Oceanic Technol., 15, 1361-1378, https://doi.org/ 10.1175/1520-0426(1998)015<1361:DOORRA>2.0.CO;2.

Rainwater, S., C. H. Bishop, and W. F. Campbell, 2015: The benefits of correlated observation errors for small scales. Quart. J. Roy. Meteor. Soc., 141, 3439-3445, https://doi.org/10.1002/ qj.2582.

Rio, M.-H., A. Pascual, P.-M. Poulain, M. Menna, B. Barceló, and J. Tintoré, 2014: Computation of a new mean dynamic topography for the Mediterranean Sea from model outputs, altimeter measurements and oceanographic in situ data. Ocean Sci., 10, 731-744, https://doi.org/10.5194/os-10-731-2014.

Ruggiero, G. A., E. Cosme, J. Brankart, J. Le Sommer, and C. Ubelmann, 2016: An efficient way to account for observation error correlations in the assimilation of data from the future SWOT high-resolution altimeter mission. J. Atmos. Oceanic Technol., 33, 2755-2768, https://doi.org/10.1175/ JTECH-D-16-0048.1.
Stammer, D., 1997: Global characteristics of ocean variability estimated from regional TOPEX/Poseidon altimeter measurements. J. Phys. Oceanogr., 27, 1743-1769, https://doi.org/ 10.1175/1520-0485(1997)027<1743:GCOOVE > 2.0.CO;2.

_ tropic ocean tide models. Rev. Geophys., 52, 243-282, https:// doi.org/10.1002/2014RG000450.

Stewart, L. M., S. L. Dance, and N. K. Nichols, 2013: Data assimilation with correlated observation errors: Experiments with a 1-D shallow water model. Tellus, 65A, 19546, https://doi.org/ 10.3402/tellusa.v65i0.19546.

Storto, A., 2016: Variational quality control of hydrographic profile data with non-Gaussian errors for global ocean variational data assimilation systems. Ocean Modell., 104, 226-241, https://doi.org/10.1016/j.ocemod.2016.06.011.

__ S. Dobricic, S. Masina, and P. Di Pietro, 2011: Assimilating along-track altimetric observations through local hydrostatic adjustment in a global ocean variational assimilation system. Mon. Wea. Rev., 139, 738-754, https://doi.org/ 10.1175/2010MWR3350.1.

_ - S. Masina, and S. Dobricic, 2013: Ensemble spread-based assessment of observation impact: Application to a global ocean analysis system. Quart. J. Roy. Meteor. Soc., 139, 1842 1862, https://doi.org/10.1002/qj.2071.

__ , and Coauthors, 2017: Steric sea level variability (1993-2010) in an ensemble of ocean reanalyses and objective analyses. Climate Dyn., 49, 709-729, https://doi.org/10.1007/s00382-0152554-9.

Taburet, G., and Coauthors, 2018: Sea level TAC-DUACS products. Copernicus Marine Environment Monitoring Service Doc. CMEMS-SL-QUID-008-032-051, 66 pp., http:// cmems-resources.cls.fr/documents/QUID/CMEMS-SL-QUID008-032-051.pdf.

Verrier, S., P.-Y. Le Traon, and E. Remy, 2017: Assessing the impact of multiple altimeter missions and Argo in a global eddy-permitting data assimilation system. Ocean Sci., 13, 1077-1092, https://doi.org/10.5194/os-13-1077-2017.

Vidard, A., M. Balmaseda, and D. Anderson, 2009: Assimilation of altimeter data in the ECMWF ocean analysis system 3. Mon. Wea. Rev., 137, 1393-1408, https://doi.org/10.1175/ 2008MWR2668.1.

Waller, J. A., S. L. Dance, and N. K. Nichols, 2016: Theoretical insight into diagnosing observation error correlations using observation-minus-background and observation-minusanalysis statistics. Quart. J. Roy. Meteor. Soc., 142, 418-431, https://doi.org/10.1002/qj.2661.

Weatherall, P., and Coauthors, 2015: A new digital bathymetric model of the world's oceans. Earth Space Sci., 2, 331-345, https://doi.org/10.1002/2015EA000107.

Weaver, A. T., C. Deltel, E. Machu, S. Ricci, and N. Daget, 2005: A multivariate balance operator for variational ocean data assimilation. Quart. J. Roy. Meteor. Soc., 131, 3605-3625, https:// doi.org/10.1256/qj.05.119.

Weston, P., 2011. Progress towards the implementation of correlated observation errors in 4D-Var. Met Office Tech. Rep. $560,33 \mathrm{pp}$.

Wunsch, C., and D. Stammer, 1998: Satellite altimetry, the marine geoid, and the oceanic circulation. Annu. Rev. Earth Planet. Sci., 26, 219-253, https://doi.org/10.1146/annurev.earth.26.1.219.

Yaremchuk, M., J. M. D’Addezio, G. Panteleev, and G. Jacobs, 2018: On the approximation of the inverse error covariances of high-resolution satellite altimetry data. Quart. J. Roy. Meteor. Soc., 144, 1995-2000, https://doi.org/10.1002/qj.3336. 